\title{
"EL LIBRO QUE CONTIENE ONZE PARTIDOS DEL M. DN JOSEPH DE TORRES”. ¿TORRES Y MARTÍNEZ BRAVO, O TORRES Y VERGARA? APROXIMACIONES A UNA INTERPRETACIÓN FILOLÓGICA DEL MANUSCRITO DE LA COLECCIÓN SÁNCHEZ GARZA*
}

\author{
Gustavo Delgado Parra \\ Universidad Nacional Autónoma de México
}

Resumen:

El Libro de Torres de la colección Sánchez Garza, es uno de los documentos más polémicos de los últimos tiempos en la musicología hispánica. Esta polémica ha sido el resultado de dos factores fundamentales: por un lado el problema de la autoría, y por el otro, el aparente desorden del contenido musical del manuscrito, debido supuestamente, al trabajo ineficiente de los copistas que le dieron forma. Esto último ha llevado a concluir, como opinión generalizada, que se trata de una copia corrompida de las obras para órgano que habrían sido compuestas por el prestigiado compositor, organista, teórico musical e impresor español, Joseph de Torres y Martínez Bravo (*1670c; †1738), en cuyo caso el copista del manuscrito sería el responsable de dicho desorden; lo que ha llevado a suponer, a otros investigadores, que incluso las obras en cuestión no sean de su autoría. A esto debemos agregar la confusa y a veces errónea transcripción ofrecida por el CENIDIM en $1993^{1}$, en cuyo caso las obras han sido adjudicadas a José de Torres y Vergara, un personaje mexicano, homónimo y contemporáneo de Torres y Martínez Bravo.

Palabras clave:

Torres, Capilla Real, Música para tecla, Siglo XvIII.

\footnotetext{
* Este artículo es un apartado de mi tesis doctoral, dirigida por el Dr. Antonio Ezquerro, y defendida públicamente ante tribunal en la Universidad Politécnica de Valencia (Departamento de Comunicación Audiovisual, Documentación e Historia del Arte; Programa: "Música" con mención de calidad) el 27 de octubre de 2007, la cual se tituló Un libro didáctico del siglo xVIII para la enseñanza de la composición: el "Libro que contiene onze partidos" para órgano de Joseph de Torres en la colección “Jesús Sánchez Garza” del CENIDIM (México, D.F.). Dicho trabajo académico de investigación, obtuvo la máxima calificación (Sobresaliente cum laude -por unanimidad-), habiendo recibido, recientemente, Premio Extraordinario de Tesis Doctorales 2009 en la universidad mencionada, así como Mención única en el xi Premio de Musicología "Casa de las Américas". La presente investigación (tesis doctoral y artículo que hoy se edita) han sido convenientemente presentados en el Registro de la Propiedad Intelectual (oficina de Barcelona), con número de entrada B-2731-09, con todos los efectos legales y jurídicos que marca el derecho internacional para todos los países.

1 Ramírez Ramírez, Felipe, ed.: El libro que contiene onze obras para órgano de registros partidos del Dr. D. Joseph de Torres. México, CENIDIM, 1993.
} 


\begin{abstract}
:
The Torres Book from the Sanchez Garza collection is one of the most polemic issues in the study of Hispanic music. This controversy has been the result of two main factors. On the one hand, the authorship problems and on the other, the apparent disorder of the musical content in this manuscript due to the inefficient work done by the copiers who gave it form. The latter has lead to conclude, as a generalized opinion, that it is a highly corrupted copy of the organ works that could have been composed by the famous Spanish composer, organist, musical theorist and printer, Joseph de Torres y Martinez Bravo $(* 1670$ c; $\dagger 1738)$; in which case the copier would be the one to blame for the demerit of such a renowned musician of the Royal Chapel of Madrid. If to this we add the transcription offered by the CENIDIM in 1993 which multiplies the confusion, we are facing pieces of music full of questions and a musical text which is neither effective nor convincing.
\end{abstract}

Key Words:

Torres, Spanish Royal Chapel, Keyboard Music, 18th Century.

La primera razón para investigar este tema radica en la importancia del manuscrito del Libro de Torres y su intrínseca relación con la música antigua hispanoamericana para tecla (órgano). La música mexicana de tecla de los siglos XVII y XVIII constituye a la fecha un número muy limitado de obras; sin embargo, y pese a las circunstancias, es un importante patrimonio que forma parte de nuestra identidad cultural no menos importante a aquél de los órganos históricos, si bien este último, cuantitativamente más importante, es el testimonio fehaciente de que debió existir una importante producción de música para tecla, hoy casi extinta, o al menos, escasamente investigada. En el caso del Libro de Torres nos encontramos además con el único testimonio de lo que podrían ser las obras para órgano de Joseph de Torres y Martínez Bravo, cuya obra no es interesante sólo desde un punto de vista histórico o musicográfico, sino por su gran calidad musical. El interés creciente por la música antigua iberoamericana para órgano en general y por toda aquella música encontrada en archivos mexicanos, reflejada en la proliferación de festivales y edición de discos y partituras, con gran repercusión en el extranjero, afirma esta elección.

Otra razón, aunque hay un cierto número de trabajos publicados sobre el Libro de Torres, es que es necesaria una nueva propuesta musicológica, debido al estado de las investigaciones, de las cuales ninguna ha abordado el tema desde el análisis musical de las obras mismas, resumiéndose a especulaciones que otorgan, o quitan, de manera arbitraria, la paternidad de estas obras a José de Torres y Martínez Bravo. Algunos trabajos como el ya citado de Felipe Ramírez, influenciado por "tópicos" modernos generalizadamente aceptados, intenta analizar las obras desde un punto de vista de la armonía funcional, amén de la interpretación del ms., la cual redunda en una interpretación en varias ocasiones equívoca.

Considero que una plataforma fundamental, a partir de la cual es posible adjudicar, o suprimir la autoría de la obras contenidas en el ms. al español, José de Torres, es a través de su obra musical y teórica, de la cual, Torres nos ha legado un importante acervo. Algunos de los trabajos hasta ahora publicados se han concretado a hacer comentarios breves o especulaciones sobre la autoría de las obras contenidas en el Libro de Torres a partir de datos historiográficos aislados o por referencias de terceros, no aportando respuestas científicamente comprobables al tema. En este sentido, el presente 
estudio es el primero que aborda esta problemática desde la perspectiva de un análisis codicológico, musical y documental, ahondando en los procedimientos constructivos y las técnicas de composición empleadas en el manuscrito, en relación con la obra teórico-musical de José de Torres y Martínez Bravo. Asimismo, se analizan los aspectos paleográficos, o rasgos del manuscrito, con la intención de ofrecer nuevas lecturas del documento desde la perspectiva de la musicología histórica, abordando las fuentes documentales con rigor científico, con la intención de enriquecer las formas de difusión del patrimonio musical documental de México.

La presente investigación hace una interpretación, hasta ahora inédita, del extraordinario valor artístico, teórico e histórico de las obras para tecla de José de Torres; y despeja cualquier duda en torno a la dudosa autoría que hasta hoy había ensombrecido sus obras, contenidas en el manuscrito de la "Sánchez Garza".

Este artículo no recoge muchas de las conclusiones y reflexiones expuestas a lo largo de mi investigación, como son los relativos al proceso de aprendizaje musical en el contexto históricogeográfico que nos ocupa (dinámica del proceso de aprendizaje, correcciones localizadas en la reelaboración de los motivos empleados, posibles tratados que se tomaron como base, etc.). Estos detalles, la transcripción de las obras, una edición crítica y otros temas relevantes, son tratados con profundidad en mi tesis doctoral (UPV 2007), publicada en 2009, denominada: Un libro didáctico del siglo XVII para la enseñanza de la composición: el "Libro que contiene onze partidos" para órgano de Joseph de Torres en la colección “Jesús Sánchez Garza” (México, D.F.).

\section{Estado de la Cuestión}

\section{Cuestiones de autoría}

En primer lugar conviene precisar que la supuesta autoría de las obras contenidas en el manuscrito, así como la realización del mismo, atribuida por Felipe Ramírez al mexicano Joseph de Torres y Vergara (*1661; †1727), en la publicación del CENIDIM de $1993^{2}$, resulta por demás cuestionable, ya que hasta la fecha no se ha podido ni siquiera probar que este notable personaje del mundo novohispano, tuviera entre sus múltiples virtudes la de ser músico.

Una de las justificaciones de F. Ramírez, para eliminar el nombre de Torres y Martínez Bravo de la autoría de las obras del ms., es que algunos musicólogos españoles como: Higinio Anglés, Miguel Querol, José Subirá, Samuel Rubio y Dionisio Preciado, no dan testimonio de que este compositor tenga música para órgano ${ }^{3}$.

2 Ramírez Ramírez, Felipe: El libro que contiene..., op. cit.

3 Ramírez Ramírez, Felipe: El libro que contiene..., op. cit., p. XII. 
Esta aseveración por parte de Ramírez ciertamente resulta insostenible, si tan solo tomamos en cuenta el hecho de que, algunos contemporáneos suyos, a pesar de no profesar en algunos casos particular empatía por José de Torres, reconocían su merito como organista. Tal es el caso del testimonio de Vicente Pérez, Tenor de la Capilla Real, emitido 13 años después de la muerte de Torres:

No obstante la ponderación que aquí hace D. Josef Nebra del Maestro D. Josef Torres, entiéndase que si tubo mérito particular podría le tubiere en el órgano cuando era organista $[\ldots]^{4}$.

Guillermo Tovar y de Teresa señala que el Dr. Joseph de Torres y Vergara fue heredero de una importante fortuna por parte de Don Juan Caballero y Ocio $(\dagger 1707)^{5}$, y que con esos recursos Torres y Vergara realizó obras tales como el retablo de la Capilla de los Arcángeles de la Catedral Metropolitana de México (llevado a cabo hacia 1712 por el escultor Manuel de Nava) y un sinnúmero de obras piadosas ${ }^{6}$.

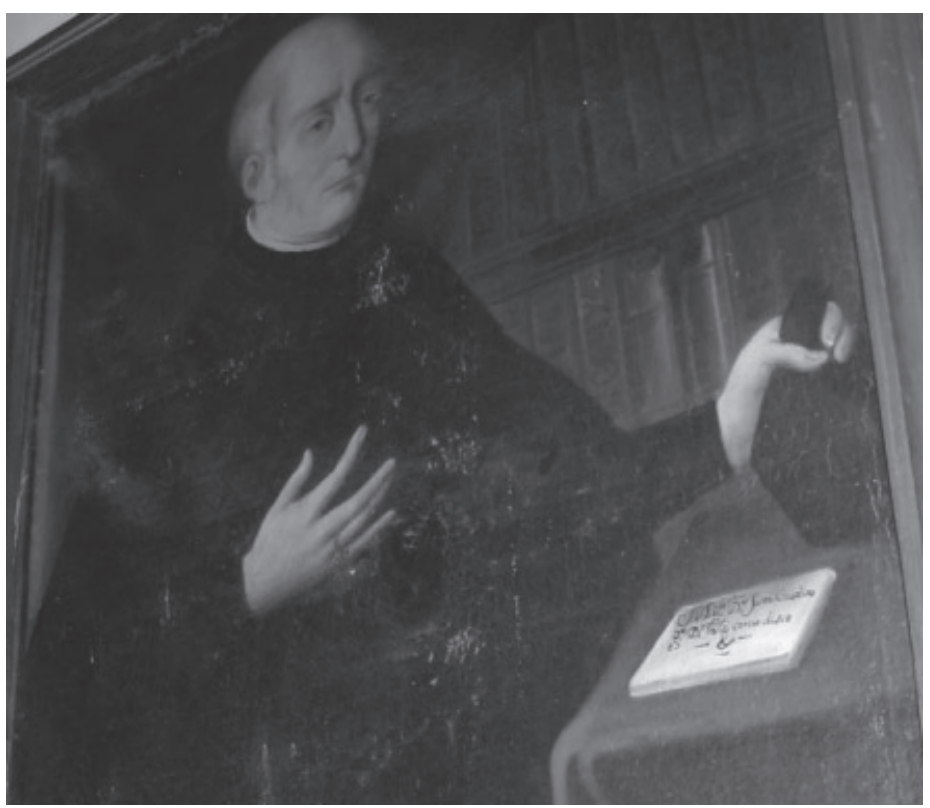

Cuadro al óleo de Don Juan Caballero y Ocio ${ }^{7}$

4 Pérez, Vicente: Libro de constituciones y ordenanzas de la Real Capilla de su S.M. Madrid, 1791. Memorial de fecha "Madrid y Junio de 1751". [Ms. No 64].

5 Caballero y Ocio, sacerdote y benefactor, patrocinó diversas obras arquitectónicas en la ciudad de Querétaro y sus donativos a las Misiones de California le procuraron el titulo de "Adelantado de las Californias", honor al cual renunció.

6 Beristain De Souza, José Mariano: Biblioteca Hispanoamericana Septentrional. México, 1821, T. III, p. 125. Editada por el Instituto de Estudios y Documentos Históricos, A.C., Claustro de Sor Juana, México, 1980 y, Universidad Nacional Autónoma de México, 1981.

7 El cuadro, de autor anónimo, se encuentra en la iglesia de la Congregación de Querétaro, México. Foto del autor. 
Así pues, tanto Caballero y Ocio, como Torres y Vergara, son personajes conocidos y representativos del clero, que promovieron y patrocinaron la creación de obras artísticas.

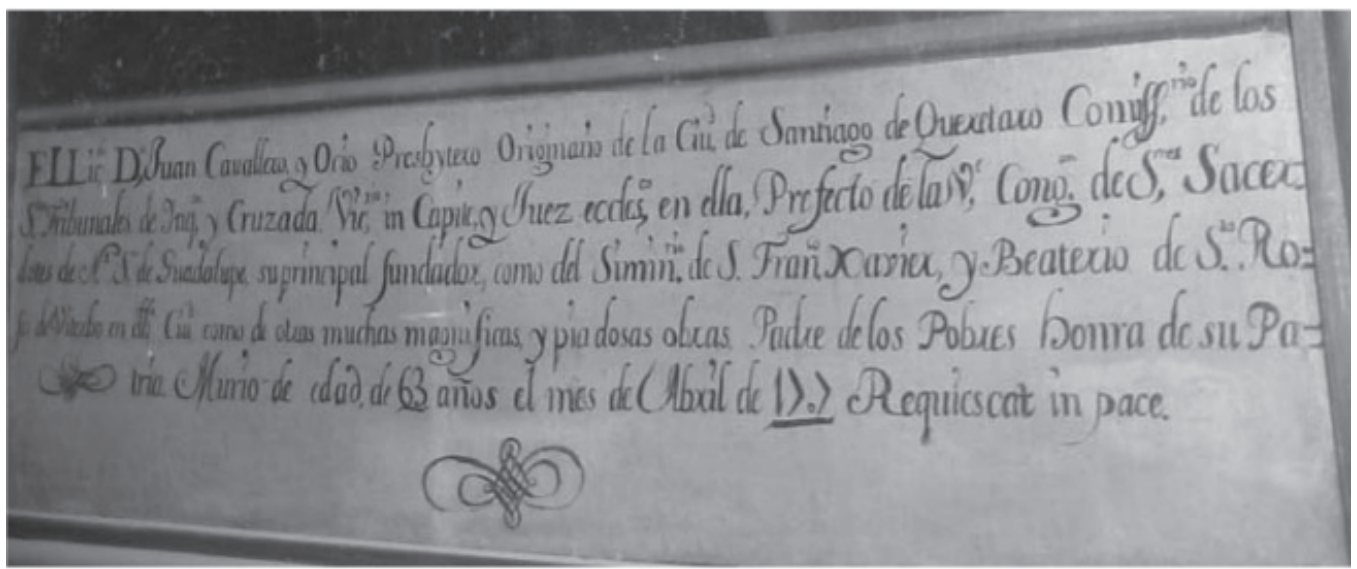

Detalle del cuadro de Don Juan Caballero y Ocio, en el que se menciona su origen, cargos, así como la fecha de su muerte, acaecida el año de $1707^{8}$.

Por otro lado, Torres y Vergara también fue jurista. En la Real y Pontificia Universidad, fue catedrático y decano, abogado en la Audiencia y consultor de la Inquisición. Como jurista, produjo dos libros impresos en México, uno en 1722 y otro en 1727; se trata de alegatos: una respuesta jurídica y una disertación canónica moral sobre el derecho de acrecer de prebendados enfermos según la costumbre del Cabildo de la Catedral. En este aspecto el Dr. Joseph de Torres y Vergara fue funcionario eclesiástico y teórico en Derecho Canónico, cuya carrera en el Cabildo culminó con el cargo de Arcediano. Fue impulsor de la vida musical de la Catedral Metropolitana. Fue electo Chantre en los años en que floreció la Capilla musical con Antonio de $\operatorname{Salazar}^{9}(* 1650$ c; $\uparrow 1725$ c) y Manuel de Sumaya $(* 1684 c ; \nmid 21.12 .1755)^{10}$. Indudablemente, estas noticias relacionan al Dr. Joseph de Torres y Vergara con la vida musical de la Catedral de México ${ }^{11}$. No obstante, y aún en el caso de que Joseph de Torres y Vergara hubiese tenido algún nexo con el quehacer de la composición musical, no existe evidencia alguna en este campo, de su obra, a través de la cual pudiéramos cotejar y encontrar paralelismos que permitieran especular sobre su autoría del manuscrito en cuestión. Más aún, el presente estudio prueba con evidencias suficientes, como se podrá comprobar, que esa postura es totalmente arbitraria.

8 Idem.

9 Antonio de Salazar. Organista, bajonero y compositor, nace probablemente en Puebla hacia 1650. Entre 1672 y 1688 , tuvo el cargo, ganado por oposición, de Maestro de Capilla de la Catedral de Puebla. El 26 de agosto de 1688 fue elegido, también por oposición, Maestro de Capilla de la Catedral de México. Entre sus alumnos cabe destacar a Manuel de Sumaya.

10 Estudió con Antonio de Salazar y Joseph de Idiaques. En 1708 se le nombró segundo organista de la catedral de México, y posteriormente sucedió a Joseph de Idiaques como primer organista. En 1711 fue nombrado segundo Maestro de Capilla y cuatro años después, en 1715, alcanzó el puesto de primero, a la muerte de Antonio de Salazar. En 1739 se traslada a Antequera (hoy cd. de Oaxaca) en donde permanece al servicio de la Catedral de Oaxaca, hasta su muerte acaecida el 21 de diciembre de 1755.

11 Tovar y De Teresa, Guillermo: "Proemio", en El libro que contiene onze..., op. cit. 1993, pp. IX - XII. 
Cabe señalar la existencia de un retrato al óleo, de autor anónimo, del Dr. Don Joseph de Torres y Vergara, actualmente en el Museo Nacional del Virreinato (en Tepotzotlán, Estado de México). Este cuadro es tomado, entre otros documentos, por Felipe Ramírez como testimonio de "su responsabilidad [de Torres y Vergara] en el arte musical”. La leyenda al calce del cuadro dice lo siguiente:

El Sr. Dr. D. José de Torres y Vergara, Arcediano de esta Santa Iglesia, Cancelario de la Rl. [Real] Universidad, Juez revisor de los naturales de Capellanías y Obras Pías de este Arzobispado Consultor de el Santo Oficio. Capellán de las Sras. Relsas. Carmelitas de Teresa la Antigüa. Y dio para este Colegio 10,000 ps. Méjico Septiembre 14 de 1725.

Tal como puede leerse en la leyenda, y pese a lo detallado de la descripción de algunos de los numerosos cargos ejercidos por Torres y Vergara, no hay referencia alguna que lo ratifique como compositor, o a su quehacer en el campo de la música. Otra cosa es que entre sus obras pías haya incluido, por ejemplo, la fundación del Colegio de Infantes para los niños del Coro de la Catedral de México en 1726. Sin embargo, esas prerrogativas no le confieren crédito alguno como músico.

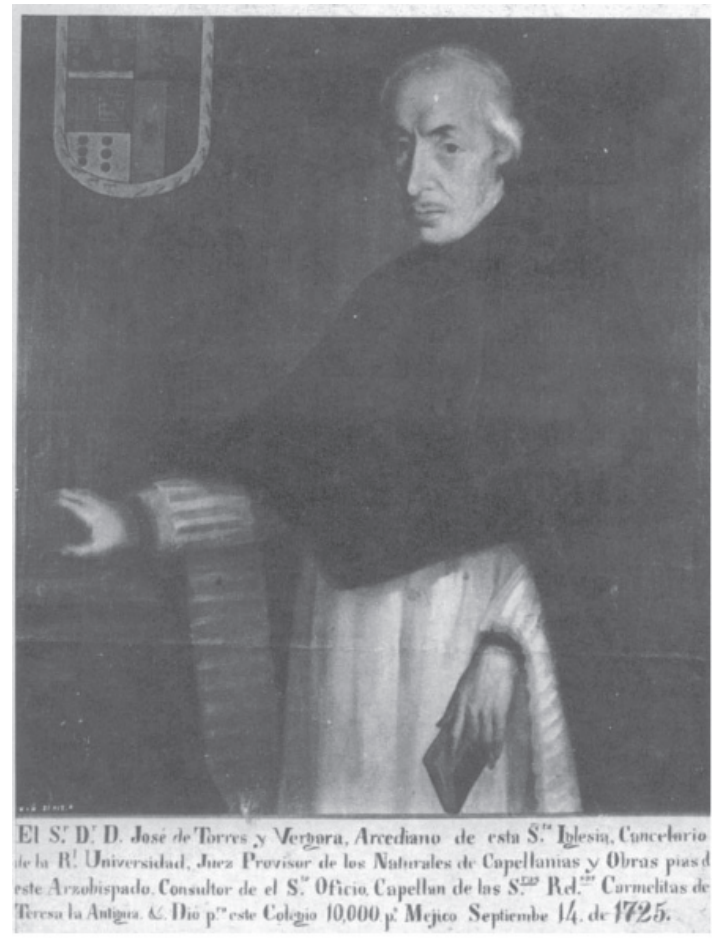

Pintura del Sr. Dr. Dn. José de Torres y Vergara ${ }^{12}$

12 Obra anónima, actualmente en el Museo Nacional del Virreynato. 
Por el contrario, el español Joseph de Torres y Martínez Bravo es un personaje cuya vida y obra musical está ampliamente documentada, y a partir de la cual podemos constatar nexos entre su obra y el ms. que nos ocupa. Por todo ello, y a partir de este momento, al mencionar a Joseph de Torres en este trabajo, me referiré al español Joseph de Torres y Martínez Bravo (*1670c; †1738). Vale la pena adelantar, sin embargo, que las obras de esta colección, en algunos detalles, no son íntegramente de su autoría.

\section{EL MANUSCRITO}

\section{BREVE ANÁLISIS CODICOLÓGICO}

\section{Ubicación y características del manuscrito}

El manuscrito forma parte de la Colección Sánchez Garza ${ }^{13}$, actualmente en custodia del Centro Nacional de Investigación, Documentación e Investigación Musical (CENIDIM) ${ }^{14}$. La colección fue reunida por Jesús Sánchez Garza ${ }^{15}$ a lo largo de su vida y es una de las colecciones más importantes, tanto cualitativa como cuantitativamente, de música virreinal novohispana de los siglos XVII y XVIII ${ }^{16}$. Procede del Convento de la Santísima Trinidad de la ciudad de Puebla.

El convento de La Trinidad desapareció, sin embargo, la iglesia aún existe. Dentro de esta colección, se encuentra el manuscrito denominado: Libro que contiene onze partidos del M. Dn. Joseph de Torres. Se trata de un documento excepcional dada la inexistencia casi total de documentos de música para tecla del siglo XVII y XVIII en archivos mexicanos y, en concreto, de aquellos dedicados a la música de órgano. El ms. comprende 18 folios numerados, aparte de un folio no numerado entre los Fols. 10 y 11, el cual contiene información muy importante pese a no ser esta evidente a prima vista. Los folios tienen una medida de 21.05 x 31.0 centímetros, cada uno, y están unidos por una costura de nueve puntadas.

13 Colección Jesús Sánchez Garza:

Origen: adquirida por el Instituto Nacional de Bellas Artes (México) en 1968.

Fechas extremas: siglos XVI - XVIII.

Contenido: manuscritos novohispanos sobre todo de origen litúrgico (obras completas e incompletas).

Formado por: 19 cajas de polipropileno: 250 obras aproximadamente, descontaminadas y preservadas en guardas de polipropileno.

Nivel de organización: catalogada. Catálogo, estudio y apéndices en revisión. En proceso de asignación de signaturas.

14 El CENIDIM, fue fundado en 1974 por Decreto Presidencial, Carmen Sordo Sodi, su primera directora, había propuesto su creación un año antes —el 16 de agosto de 1973-, durante el Congreso Nacional Extraordinario de Música celebrado en la ciudad de México. A partir de entonces la misión del Cenidim, heredada en gran medida de la Sección de Investigaciones Musicales, es el conocimiento de las músicas de México y su rescate, conservación y divulgación.

15 Jesús Sánchez Garza (* Piedras Negras, Coah., 14.10.1891; †Cd. de México, 25.08.1961). Erudito e historiador. Fundador de la Sociedad Numismática de México. Autor de ensayos sobre la guerra de Texas. Coleccionista de documentos históricos, entre los cuales cabe destacar un grupo de manuscritos musicales antiguos, bautizados como Colección Sánchez Garza.

16 La colección contiene 276 manuscritos de compositores mexicanos, portugueses, españoles e italianos y anónimos del siglo XVI, XVII y XVIII. 

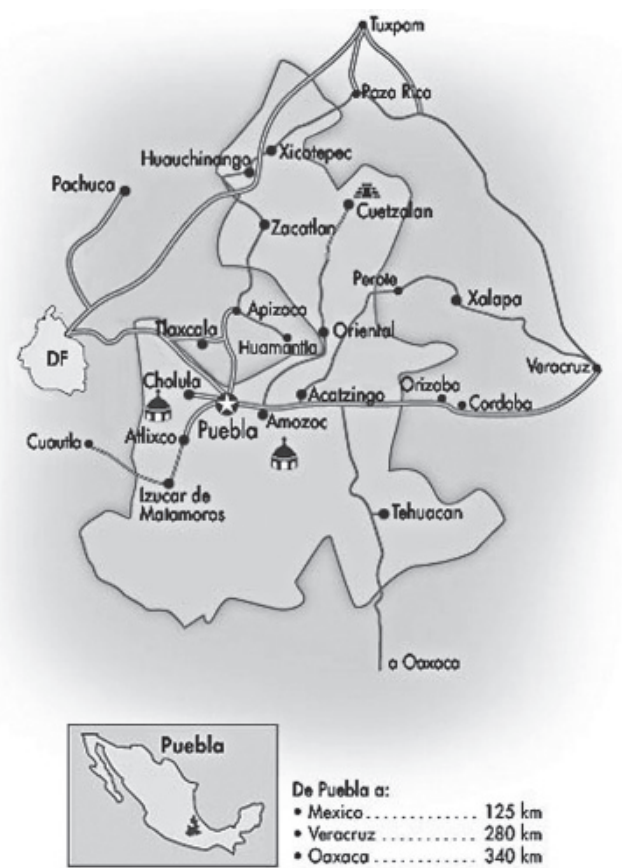

Localización geográfica del Estado de Puebla y sus colindancias con los estados vecinos. En la gráfica se puede apreciar la riqueza de órganos que posee este estado, el más rico en cuanto a población de órganos históricos se refiere en el país.

La disposición gráfica del manuscrito corresponde a la que con mayor frecuencia se encuentra en los trabajos musicales del siglo XVIII: distribución de las voces sobre dos pentagramas con alternancia de claves de Sol, Do y Fa, según las necesidades derivadas de las extensiones de las voces. Cada página contiene cuatro dobles sistemas de líneas.

El manuscrito define con toda claridad su título:

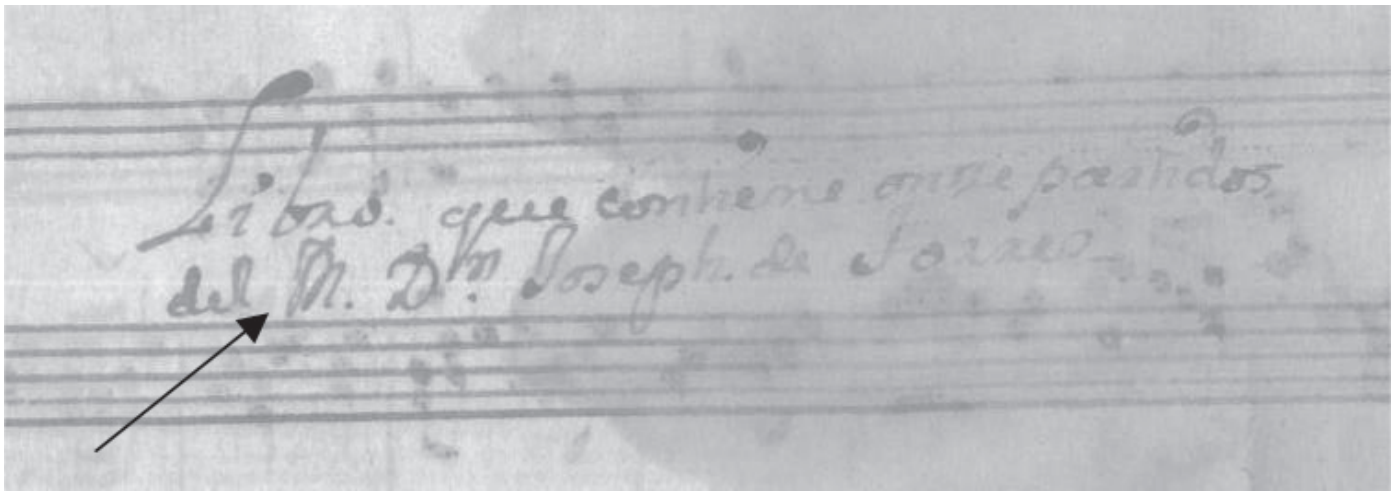

Título diplomático de la portada (folio sin numerar):

"Libro que contiene onze partidos / del M. D". Joseph de Torres" 
No hay razón, por tanto para modificarlo, agregando "Obras para Órgano”, y mucho menos para agregar el título de "Dr." al autor de estas obras, tal y como aparece en el título en la edición de Felipe Ramírez. Es evidente pues que Ramírez pretende reforzar su hipótesis a favor de Torres y Vergara interpretando e intercambiando la abreviatura Dn. por la de Dr., ya que justamente es a Joseph de Torres y Vergara a quien se le conoció como Doctor a diferencia de Martínez Bravo, como puede inferirse de la siguiente cita tomada del Manual del Viajero en Méjico o Compendio de Historia de la Ciudad de México ${ }^{17}$ :

78

MNOAL

El Colegio do Infantes para niños de coro fá fondado en 1726 por el señor arcediano Dr. D. Josed do Torres y Vergara. El 2 de febrero de 1730 vistieron por primera vez el manto de grana y la beca azal los 16 infontes músicos de la casa que está bajo la advocacion de Nuestra Señora de la Asuncion.

Detalle de la p. 78 del Manual del Viajero en Méjico de Marcos Arróniz.

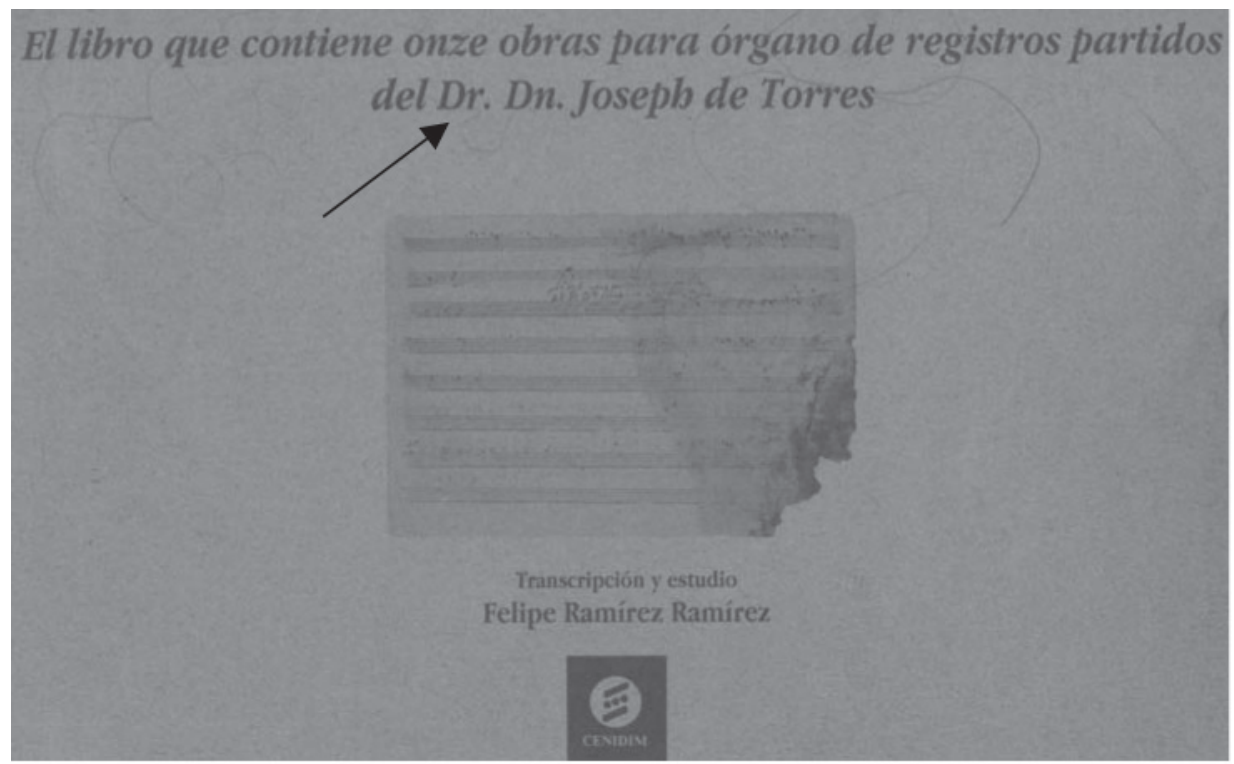

Portada de la edición del CENIDIM.

17 Arróniz, Marcos: Manual del Viajero en Méjico Compendio de Historia de la Ciudad de Méjico. París, Librería de Rosa y Bouret, 1858, p. 78. 
El número de obras en el título de la colección es de once. En la edición de Ramírez, la pieza número 2 es dividida en dos obras, cuando se trata de una sola, y con la intención de completar las once obras enunciadas en el título del manuscrito. La realidad es muy diferente: el documento solo conserva diez obras, de las cuales la núm.10 está inconclusa a causa de la pérdida de las últimas hojas del manuscrito, dentro de las cuales muy posiblemente debió estar el final de la pieza 10, y, probablemente, la pieza once y última del manuscrito.

\section{Contenidos de acuerdo a la edición de Ramírez: Contenidos de acuerdo a mi versión:}

I- Fuga I- Fuga

II- $\quad$ Obra de mano derecha de medio registro de II- Obra de mano derecha de medio registro de Torres

Torres

Andante

III- Grave Alegro (Allegro)

IV- Obra de Lleno de Séptimo tono

V- Obra de Séptimo tono

VI- Obra de Primer tono bajo

VII- Partido de Primero alto

VIII- Obra de Torres

IX- Batalla de Torres

X- Partido de $2^{\circ}$ de Torres

XI- Partido de $6^{\circ}$ tono Desp. (completado por Ramírez)
Despa $^{\circ}$ - Andante - Grave - Alegro

III- Obra de Lleno de Séptimo $7^{\circ}$ tono

IV- Obra de Séptimo tono

V- Obra de Primer tono bajo

VI- Partido de Primero alto

VII- Obra de Torres

VIII- Batalla de Torres

IX- Partido de $2^{\circ}$ de Torres

$\mathrm{X}$ - $\quad$ Partido de $6^{\circ}$ tono Desp $^{\circ}$ (inconcluso)

Por otro lado, conviene mencionar que el título del manuscrito es en sí mismo impreciso, ya que enumera onze partidos, cuando no todas las obras contenidas en él son partidos. En realidad se trata de 7 partidos y 3 obras de lleno, tal y como se puede cotejar de las formas musicales utilizadas. De hecho, sólo 3 de las obras de la colección incluyen en su título el término de "partido", siendo más común la designación de "Obra".

El manuscrito ha sido restaurado recientemente para evitar el ataque de los hongos que le habían causado un importante daño a lo largo de los años. Es de particular interés el hecho de que el ms. presenta un alto grado de deterioro en el ángulo inferior derecho de todas sus páginas, siendo este justamente el punto en donde se volteaban, lo cual nos hace suponer que fue empleado con mucha frecuencia. El deterioro generado específicamente en este lugar nos demuestra que el ms. fue empleado frecuentemente.

\section{COMENTARIOS CRÍTICOS A LA EDICIÓN DEL CENIDIM}

Considero una obligación académica el no pasar por alto al menos algunos comentarios sobre la primera edición del Libro de Torres, gracias a la cual, los investigadores, músicos y público en general, hemos tenido la oportunidad de acceder a estas obras. Sin lugar a dudas, el aporte más importante de la edición del CENIDIM ha sido el incluir en su edición el facsímil del manuscrito. 
Desafortunadamente, la edición del CENIDIM no ha hecho sino multiplicar la confusión en torno al manuscrito, debido a que la transcripción ofrecida por Felipe Ramírez tergiversa sustancialmente su contenido. El marco de referencia de mi comentario se sitúa estrictamente dentro de parámetros comprobables, dejando de lado cualquier tipo de especulación. Por ello me limito a puntualizar los siguientes casos, que por sus características ejemplifican con claridad la interpretación a todas luces errónea, y la lectura equivoca del manuscrito realizada por el transcriptor.

Caso I, “Batalla de Torres" (sexta parte, Despa'. cc. 140 a 143 del manuscrito).

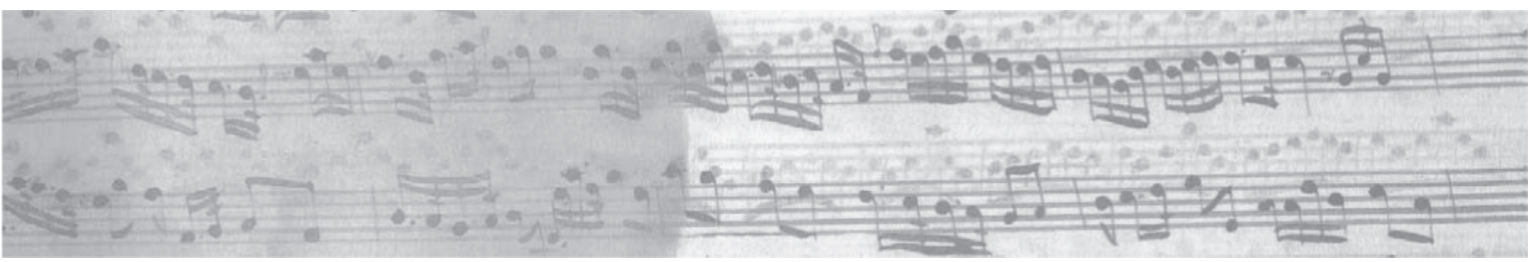

"Batalla de Torres" cc. 140 - 143, del manuscrito

La edición de Felipe Ramírez presenta la siguiente propuesta de transcripción para los cc. 140 a 143.

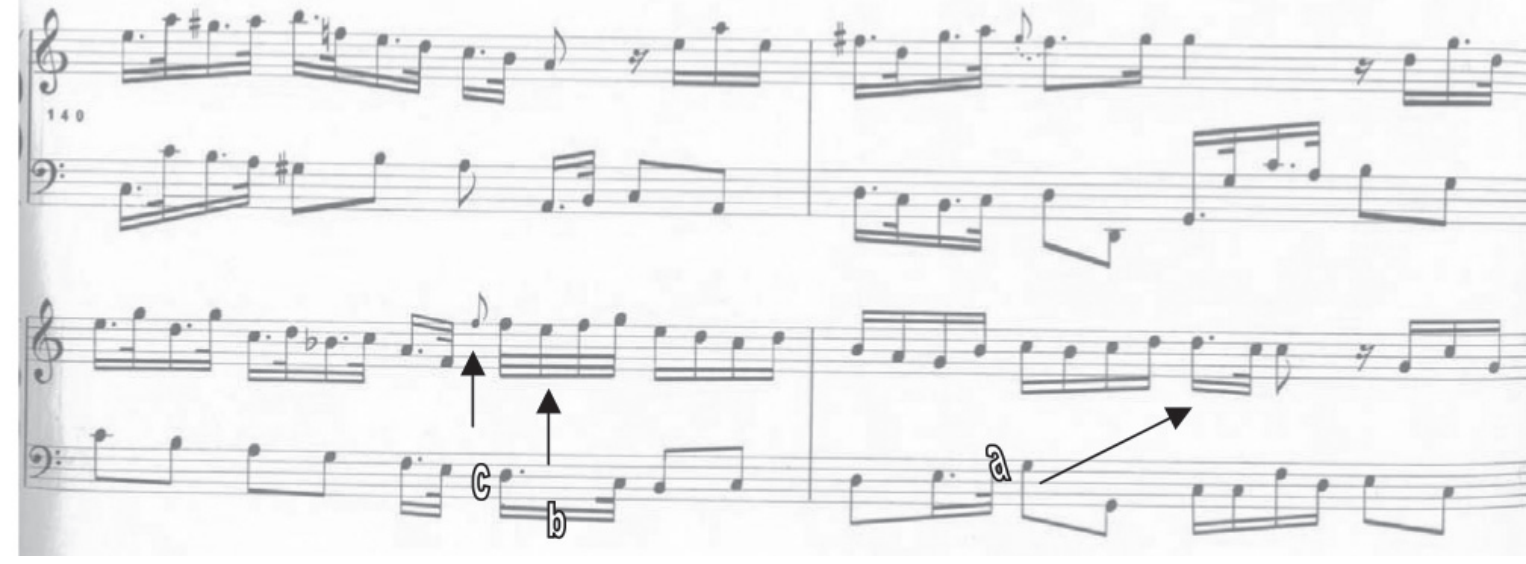

c. 142

c. 143

Como puede observarse en el ejemplo anterior, en esta interpretación hay un desfase de la estructura armónica entre la mano derecha y la izquierda a partir del segundo y tercer tiempo del c. 143 (letra a). Analizando el manuscrito podemos ver que al transcriptor le sobra medio tiempo en el c. 142 en la voz de Tiple, de manera que decide agregar un corchete al grupo de cuatro semicorcheas ubicadas en el tercer tiempo (letra b), haciéndolas fusas para contrarrestar el medio tiempo sobrante, creyendo de esta manera haber resuelto el c. 143. El desfase a partir del segundo tiempo del c. 143 es simplemente ignorado. 
De inicio, el transcriptor hace una lectura errónea del c. 142 al momento de interpretar la nota Fa aguda en el tercer tiempo como una apoyatura (acciacatura) y no como una nota real (letra c); la errónea interpretación se produce a causa de que en el c. 138 aparece en el manuscrito el adorno de acciacatura.

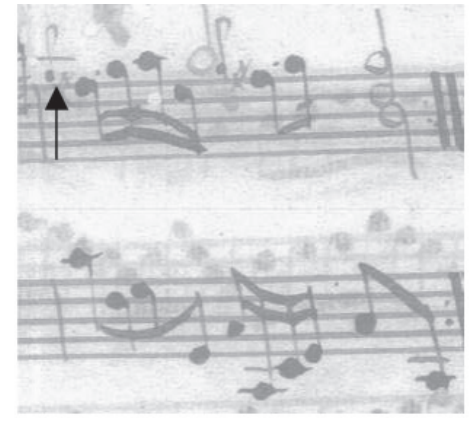

"Batalla de Torres", c 138 del ms.

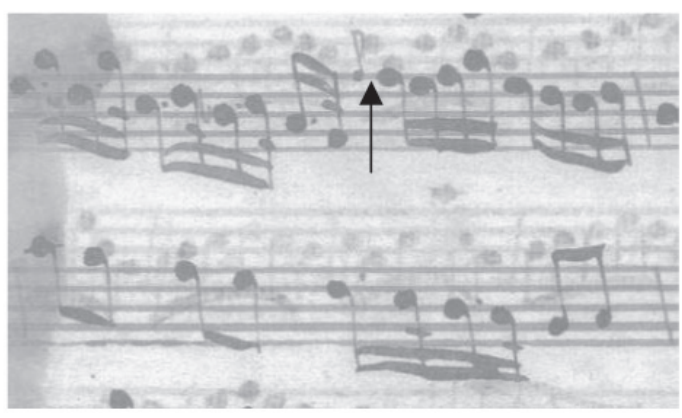

Idem, c 142

Pese a que tanto la acciacatura del c. 138 y la nota Fa aguda del c. 142 están escritas efectivamente con un tamaño más pequeño, haciéndonos pensar que esta última es también un adorno y no una nota real, no hay razón para confundir la función de cada una de ellas. Para definir esta función basta con conocer la definición de acciacatura que da Joseph de Torres en sus Reglas Generales de Acompañar:

\section{Capitulo Séptimo.}

De aquellas posturas, o golpes que llaman los italianos, Acciacaturas. "Aviendo concluido lo principal de mi assunto, me ha parecido forzoso poner aquí para mayor complemento, varias posturas, que he visto en algunos Autores Italianos, que aunque a la vista parecen muy estrabagantes, la habilidad, juycio, y destreza con que las executan las hace tolerables, y de muy rara y estraña armonia, las que regularmente practican en los Clavicordios quando acompañan aquellos golpes falsos que suele aver en los Recitados, como se demostrara adelante; siendo de advertir, que para executar, y entender dichas posturas, es preciso valerse de aquellos que llamamos mordientes, que es herir con velocidad la tecla antecedente negra, para mantenerse despues de la inmediata blanca assi que es de lo que se componen dichas posturas, o consonancias que distinguen con el nombre de Acciacatura..."

Exemplo:

E X E M P L O

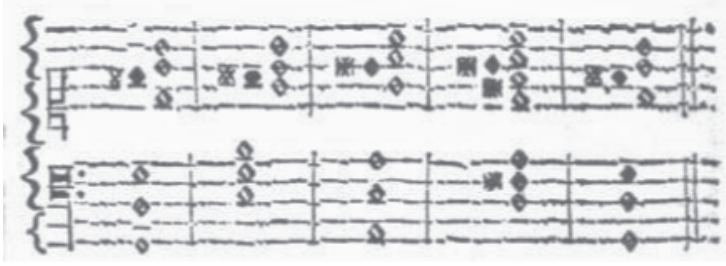


“... Advirtiendo que, para que sea más apreciables fu armonia, es de aprobado gusto, el que se entre en ellas quasi arpeando; pero no de continuo..." ${ }^{18}$.

Como se puede cotejar de la descripción de la acciacatura y de los ejemplos dados por Torres, la acciacatura es una nota cromática "extraña a la armonía" a distancia de semitono. Por lo que la interpretación del Fa agudo en cuestión queda completamente fuera de esta categoría, confirmándose como nota real.

Obsérvese que el Fa es la nueva referencia "tonal" caracterizada por el Si b tres notas antes de que se presente la nota $\mathrm{Fa}$, enfatizada a partir de la inflexión del salto de octava al registro agudo y repercutiéndose en el cuarto tiempo del c. 142, haciendo tritono, o cuarta mayor, con el Bajo para clausurar en Do.

Una vez confirmada esta nota como real no sólo nos sobraría medio tiempo en el c. 142 sino todo un tiempo. Esto significa, de hecho, que los dos grupos de semicorcheas, son en realidad fusas. Lo que se confirma plenamente a partir de la sincronización armónica resultante entre Bajo y Tiple.

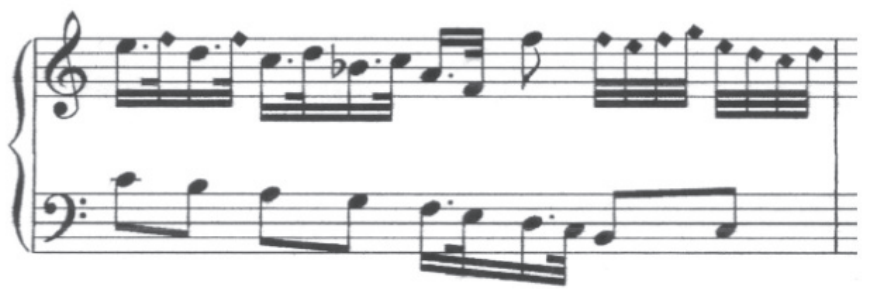

“Batalla de Torres", c. 142 corregido de acuerdo a mi versión.

Ahora bien, ¿qué sucede con el desfase armónico en el c. 143? como puede observarse en el ms., en este compás tenemos cinco tiempos en el Tiple y cuatro en el Bajo.

Ejemplo:

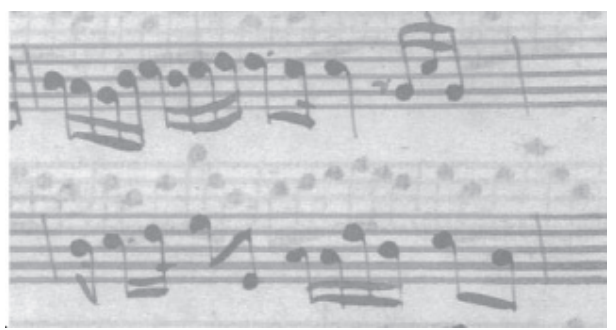

Idem, c. 143 (!) del ms.

18 Torres y Martínez Bravo, José: Reglas Generales de de acompañar, en órgano, clavicordio, y harpa, con solo saber cantar la parte, o un baxo en canto figurado. Madrid, Imprenta de Música, 1702, pp. 120-122. 
Es, pues, evidente, que nuevamente los dos grupos de semicorcheas en el Tiple son fusas y una continuación evidente de la glosa en fusas iniciada en el Tiple en el cuarto tiempo del c. 142.

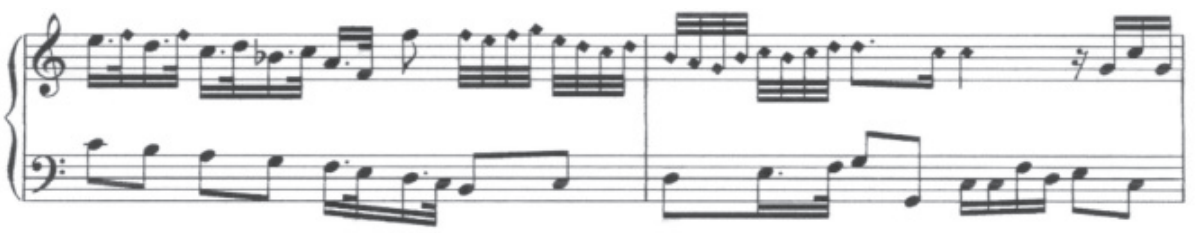

Idem, cc. 142 y 143 corregidos en mi versión.

Como puede observarse, la cuadratura armónica, rítmica y melódica, es perfecta. Cabe incluso mencionar, que la acciacatura en el c. 138 está indicada con toda claridad a través de una línea que atraviesa la nota, cosa que no sucede en la nota Fa aguda motivo de la confusión.

Como puede observarse en el manuscrito, tanto en el c. 138 como en el c. 141 aparecen unas notas pequeñas que podrían pasar por acciacaturas; sin embargo, éstas carecen de la raya atravesada que las caracteriza. Estas notas son adornos, cabezas de trinos y no son notas reales. Lo que indican es que el trino debe de empezar con la nota superior y no con la nota real, haciendo intervalo de cuarta y tomando como referencia efectivamente el concepto de acciacatura (nota extraña) aplicado a la realización de trinos. Obsérvese que en esta pieza es en la única que aparecen estas indicaciones para este tipo de adornos, lo que conlleva lo siguiente:

- Que esta manera de trinar (con la nota superior) para este tipo de trinos (trillo) era muy probablemente una "novedad" en el mundo hispánico en la primera mitad del siglo XVIII" ${ }^{19}$.

- Que la pieza VIII, "Batalla de Torres" no puede ser muy anterior a 1736, fecha en que fueron publicadas las Reglas Generales de Torres (segunda edición), ya que las influencias que dieron origen a esta pieza provienen directamente de los postulados del Tratado Quarto de ese libro, tal y como se confirma en el análisis de la pieza más adelante.

- Que podría tratarse, excepcionalmente de una de las obras para tecla tardías de Joseph de Torres y Martínez Bravo, recopilada en nuestro ms. o por lo menos se trataría de una obra muy apegada a los postulados estéticos del Torres de la "Segunda Etapa", período en el cual se deja sentir la influencia italiana en la obra de nuestro ilustre compositor.

- Que como tal, si la pieza pudo ser escrita hacia 1736, es imposible que la haya compuesto el mexicano Joseph de Torres y Vergara, muerto en 1727.

19 Hago la aclaración de que mi observación es en concreto respecto de este tipo de trinos (trillo), y no en relación con otras formas de ornamentación, tipicamente hispánicas, que empleaban la nota superior desde los siglos XVI y XVII, como por ejemplo; el Quiebro reiterado de Santa María, el cual puede ir precedido de apoyatura (nota superior), o el Quiebro de mínimas, el cual deberá practicarse solamente cuando la nota inferior a la nota adornada sea un semitono. Correa de Arauxo también consigna este adorno, pero lo denomina Quiebro reiterado. 


\section{Caso II. "Partido de $2^{\circ}$ Tono".}

En esta pieza el transcriptor ha incurrido en un caso muy similar al anterior, al no comprender la aparente falta de un tiempo en el c. 5, en la voz del Tiple, contrarrestando el tiempo que falta en el cuarto tiempo del compás, y modificando la figura rítmica de la cadencia. Ver los siguientes ejemplos:

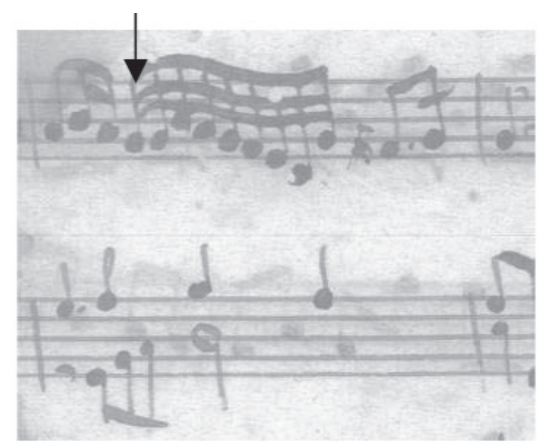

“Partido de $2^{\circ}$ tono", c. 5 del ms.

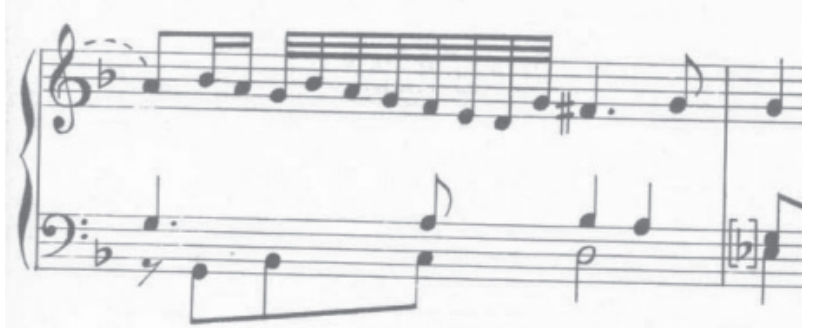

Idem, c. 5, según la trascripción de Felipe Ramírez ${ }^{20}$.

Sin embargo, la solución se revela bien distinta. Si observamos el segundo tiempo del c. 5 (Tiple) en el manuscrito, veremos que el grupo de fusas está excedido por una figura (9 figuras de fusas en lugar de 8). Esta figura excedente (nota Sol, indicada con una flecha), es la primera del grupo, en realidad se trata de una negra y no de una fusa, la cual debería estar ligada a la primera nota del grupo de fusas, de la siguiente manera:

20 Ramírez Ramírez, Felipe, ed.: El libro que contiene..., op. cit. p. 65, c. 5. 


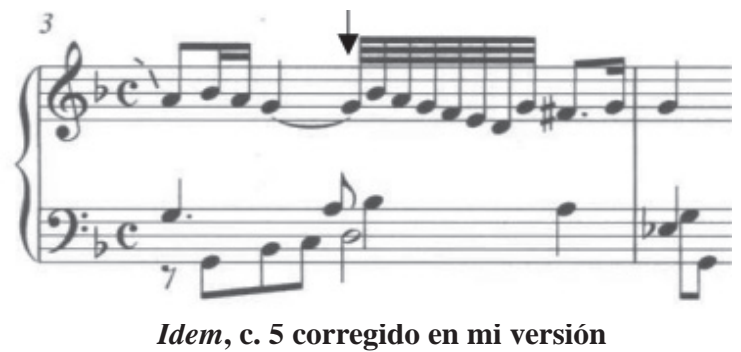

La comprobación de mi planteamiento recibe su confirmación en los cc. 8 y 9, en cuyo caso se repite exactamente la misma idea, a la quinta superior, aunque ahí desfasada a la segunda mitad del compás, tal y como aparece en el siguiente ejemplo, cc. 8, 9 .

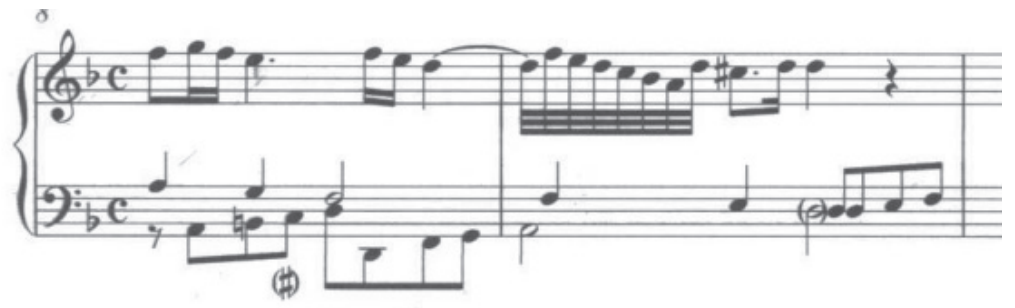

Idem, cc. 8, 9, de mi versión.

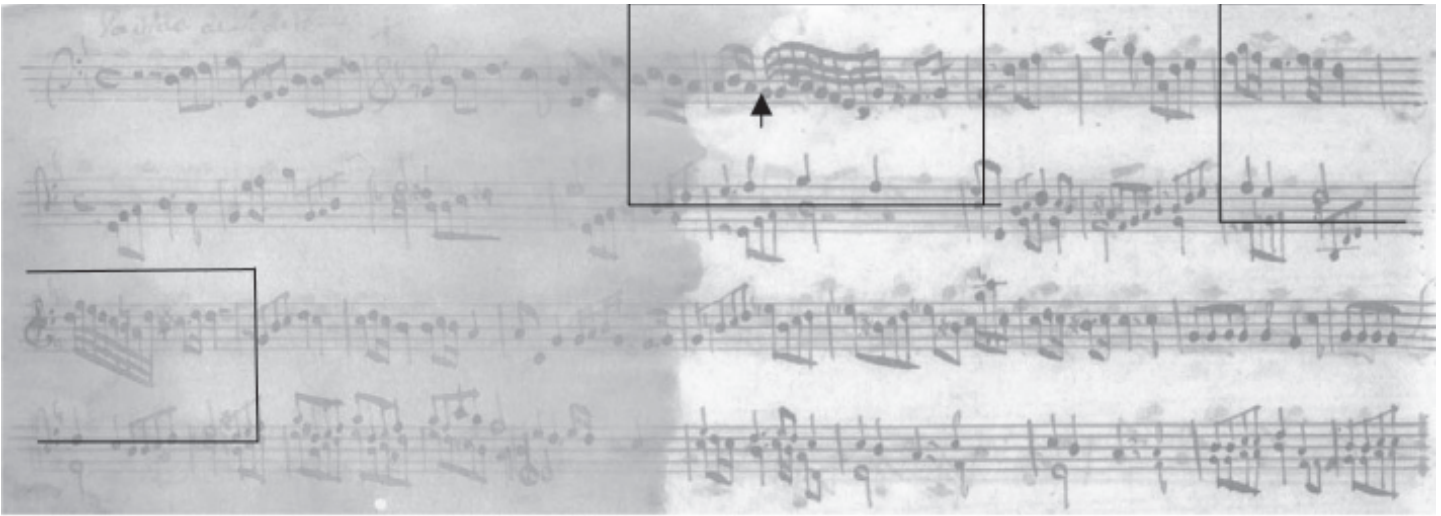

Idem, cc. 5, 8 y 9 del manuscrito.

He señalado simultáneamente, con rectángulos, las dos ideas, para que por analogía, el lector pueda ver claramente la solución de los cc. 3, 4, 5 y 6, a partir de los cc. 6, 7, 8 y 9.

Obsérvese, que a partir de la comparación ha sido posible restituir también las ligaduras de prolongación que faltan en el manuscrito (el asunto de las ligaduras es uno de los más delicados en el Libro de Torres, razón por la cual dedico más adelante todo un apartado para el análisis de este problema). 


\section{Caso III. Obra de $7^{\circ}$ Tono.}

La edición de Ramírez transcribe el primer tiempo del c. 4 de la Obra de $7^{\circ}$ Tono (en el Tenor) una segunda mayor debajo de como está escrito en el ms. Aparte escribe la mano derecha en el pentagrama inferior, a diferencia del ms. que escribe la mano derecha en el superior.

Ejemplo:

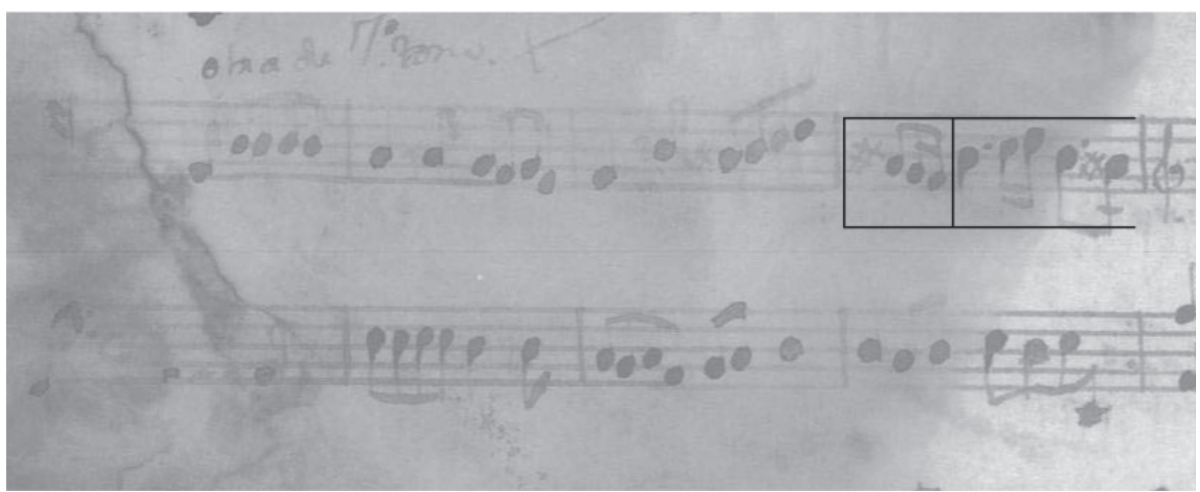

“Obra de $7^{\circ}$ tono, c. 4 del manuscrito.

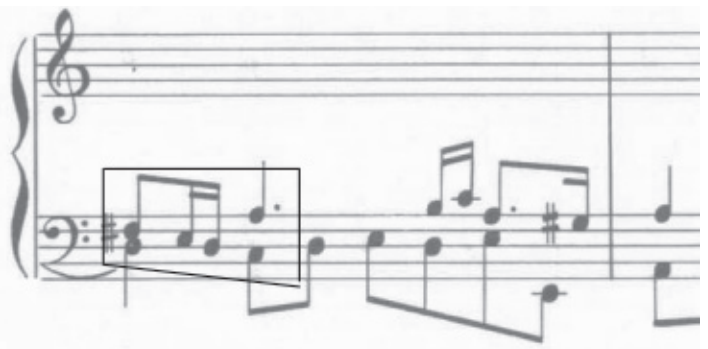

Idem, c. 4, transcripción de Ramírez ${ }^{21}$.

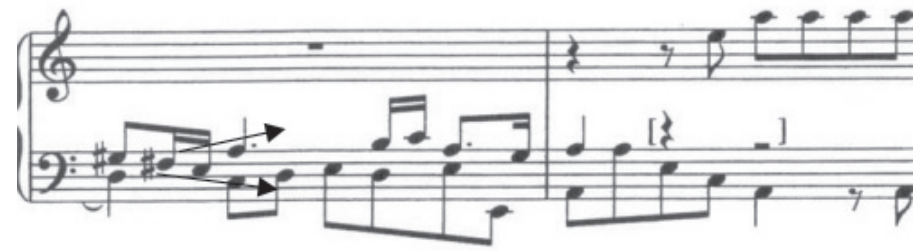

Idem, cc. 4 - 5, de mi versión.

21 RAmírez, Felipe: El libro que contiene..., op. cit., p. 22, c. 4. 
Ejemplo corregido: obsérvese la buena resolución de la Quarta mayor ( $\mathrm{Re}$ - Sol\#) por movimiento contrario.

\section{Caso IV. Obra de Lleno de $7^{\circ}$ Tono.}

El c. 14 de esta obra, en las figuras de los tiempos tres y cuatro en la mano derecha, han sido interpretadas por el transcriptor como un grupo de cuatro corcheas, cuando en el ms. está escrito como dos grupos de dos notas cada uno (bicordes en intervalo de tercera) en figuración de negras. El transcriptor ha interpretado que el copista ha omitido por descuido los corchetes y él los agrega, siendo una interpretación ciertamente errónea, tal y como puede cotejarse a continuación:

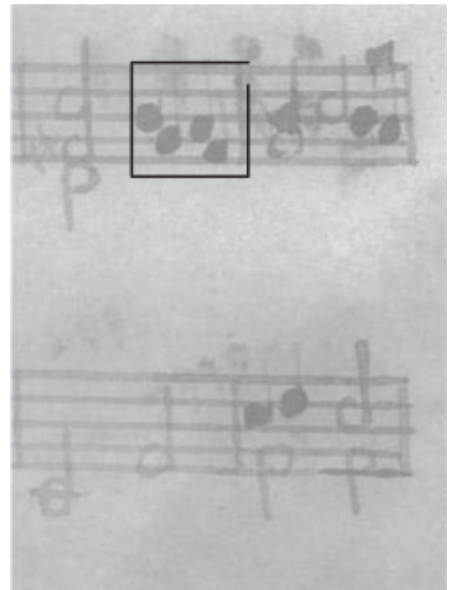

“Obra de Lleno de $7^{\circ}$ tono”, cc. 14 y 15 del manuscrito.

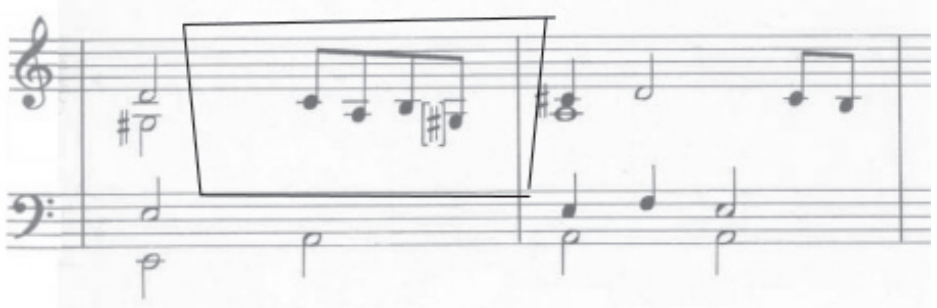

“Obra de Lleno de $7^{\circ}$ tono, cc. 14 - 15, versión de F. Ramírez ${ }^{22}$.

22 Ramírez Ramírez, Felipe: El libro que contiene..., op. cit., p. 17, c. 14. 
Prueba de ello es que, en la edición del Cenidim, se reduce en ese pasaje la textura de cuatro a dos voces, a consecuencia de interpretar esa sucesión de terceras, como terceras quebradas, alterando la textura polifónica y el color instrumental en la cadencia de una pieza construida estrictamente a 4 voces. Mi transcripción del pasaje es la siguiente:

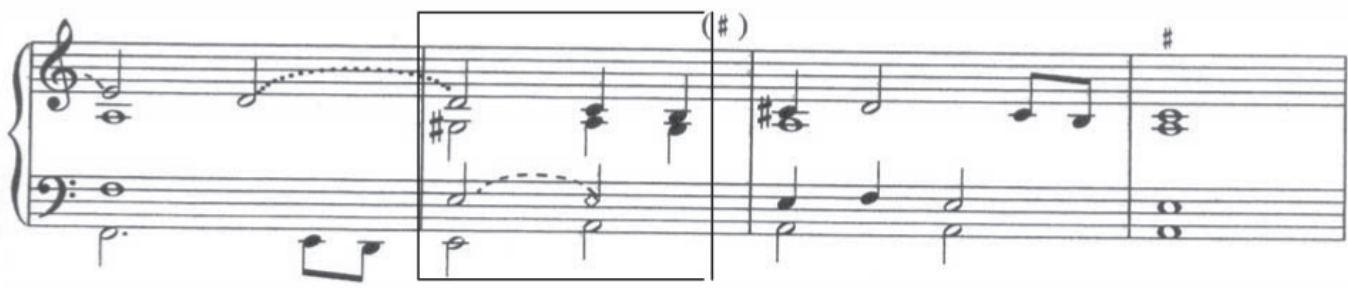

“Obra de Lleno de $7^{\circ}$ tono, cc. 13 - 16, de mi versión.

El manuscrito abunda en problemas de desfase en su forma de escritura, de hecho, Felipe Ramírez incurre en un número importante de interpretaciones erróneas, derivadas de esta forma de plasmar en el papel las ideas musicales, instrumentada por los amanuenses del Libro de Torres. La "Fuga", pieza I, es uno de los casos más claros en el cual Ramírez ratifica su visión encontrada con esas formas de caligrafía musical.

\section{Caso v. Fuga}

Este caso, parte del mismo problema que el anterior, pero llevado a un extremo (también debido a que los ejemplos son mucho menos obvios que el ya citado en la "Obra de Lleno de $7^{\circ}$ tono"), al grado de que varios pasajes de la "Fuga" llegan a transformarse en algo muy diferente de su contenido original, a manos del transcriptor. Nuevamente, el problema es la incorrecta interpretación de las figuras rítmicas, en cuyo caso, Felipe Ramírez supone que el amanuense ha omitido, por descuido, la escritura de corchetes en figuras de corcheas, o de semicorcheas; agregándolos, y modificando el contenido. No suficiente con ello, Ramírez, sin razón justificada, transcribe el segundo tiempo del c. 26 (Alto y Tiple) con notas completamente ajenas al ms., las cuales, de hecho, son absolutamente claras en el mismo. Las evidencias son las siguientes. 
Ejemplos:

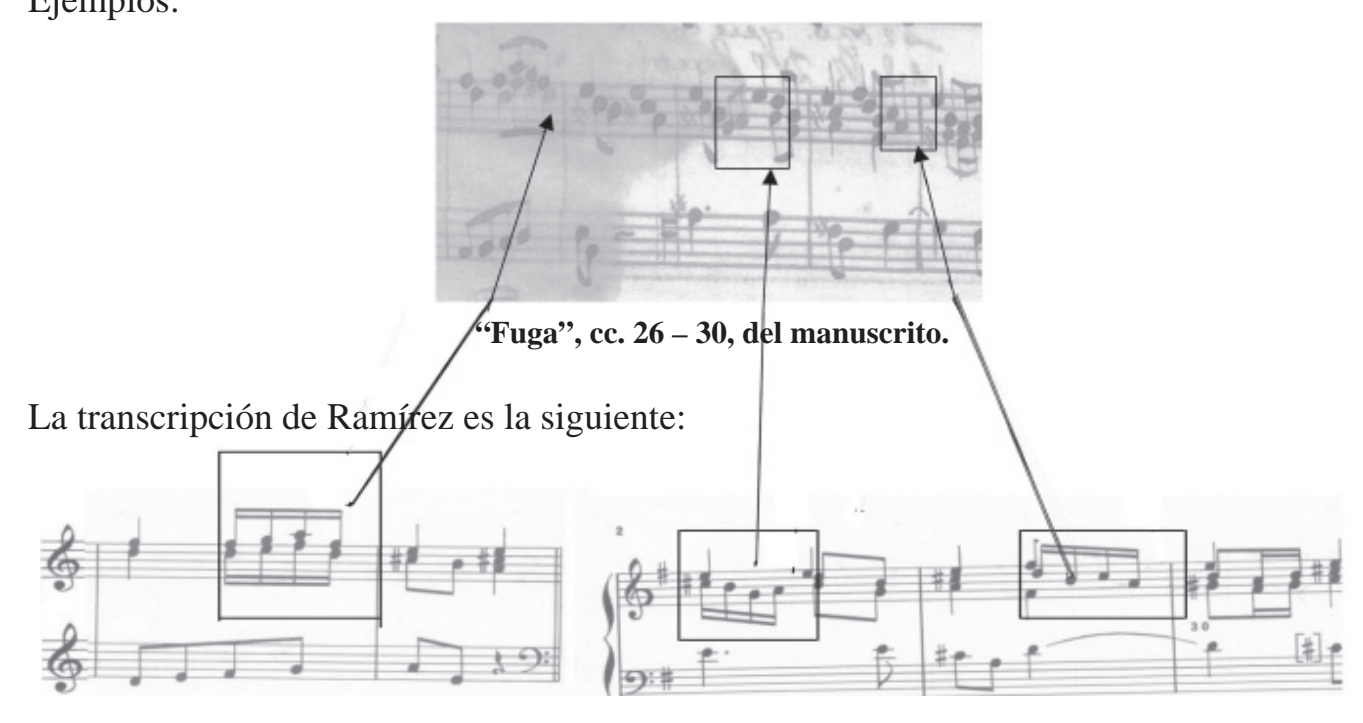

“Fuga", cc. 26 - 30, transcripción de Felipe Ramírez²3.

En realidad se trata de una escritura simultánea de acordes, y no de figuras quebradas. Si se observa, se verá que de hecho abundan los pasajes de escritura desfasada, incluso hay problemas mucho más serios de desfase a lo largo del manuscrito, (como el caso vi que se analiza enseguida). Sin embargo, Ramírez ajusta la escritura a los pasajes que no comprende, de forma que le cuadren en el compás.

Mi transcripción es la siguiente:

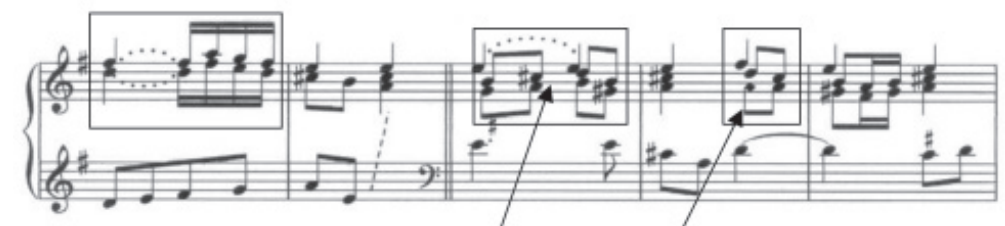

“Fuga", cc. 26 - 30, de mi transcripción.

La confirmación de mi propuesta la encontramos en el c/75 del manuscrito, en donde el pasaje es exactamente igual, pero a la quinta. Véase ejemplo:

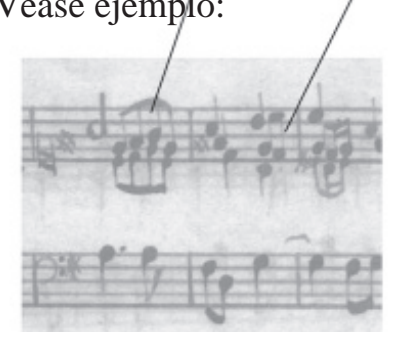

“Fuga", cc. 75 - 77, del manuscrito.

23 Ramírez Ramírez, Felipe: El libro que contiene..., op. cit., p. 1, 2, cc. 26 - 30. 
Justo un compás antes, (c. 74), el transcriptor vuelve a incurrir en los mismos problemas, al transcribir el siguiente pasaje:

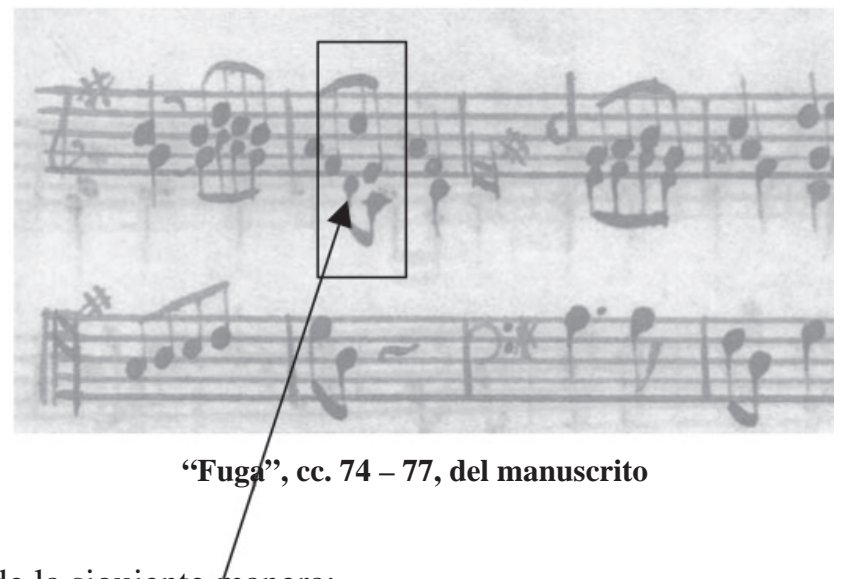

y acomodándolo de la siguiente manera:

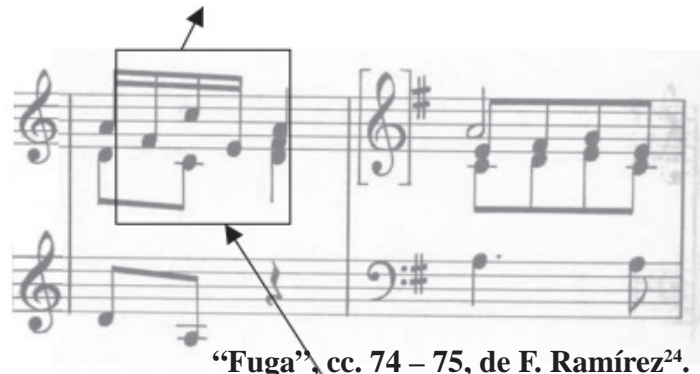

Mi transcripción es la siguiente:

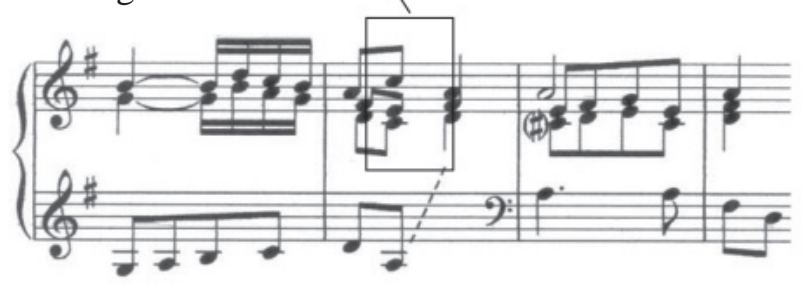

“Fuga", cc. 73 - 75, de mi versión.

24 Ramírez Ramírez, Felipe: El libro que contiene..., op. cit., p. 3, cc. 74 - 75. 
La confirmación de esta forma de escritura, se puede cotejar a través del primer ejemplo ya señalado, cc. $26-27$.

Podría continuar citando otros ejemplos como los anteriores; sin embargo, deseo sólo incluir algunos más antes de terminar este apartado, los cuales muestran a todas luces, el afán de F. Ramírez, de cuadrar las cosas a como dé lugar.

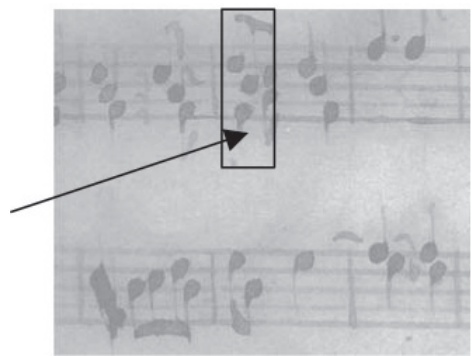

cc. 83 - 85, manuscrito.

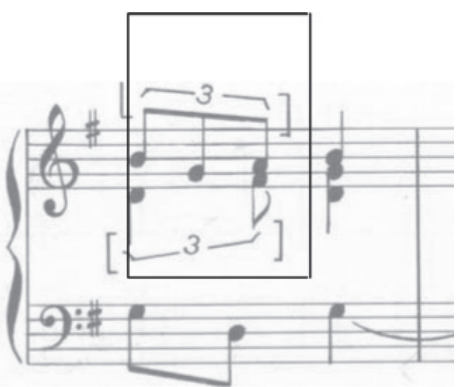

c. 84, versión de F. Ramírez

Todavía, el Mtro. Ramírez, confirma su criterio, al decir:

[...] Enlaza luego con motivos temáticos al S., e inserta un polirritmo de tres contra dos [subráyese polirritmo], iniciando una secuencia $[\ldots]^{25}$.

Mi transcripción es la siguiente:

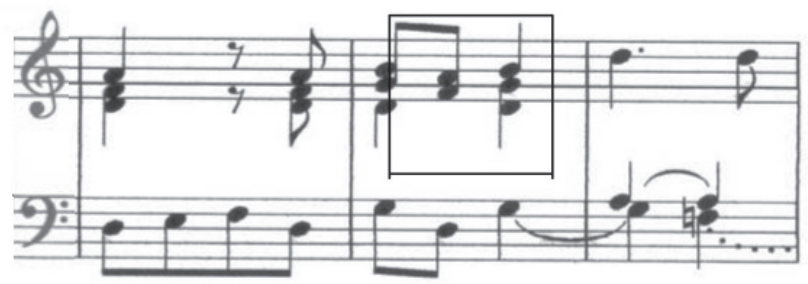

cc. 83 - 85, de mi versión.

Los otros dos casos de "polirritmia" son muy similares:

25 Ramírez Ramírez, Felipe: El libro que contiene..., op. cit., p. XVIII, Breve análisis de la obra. 


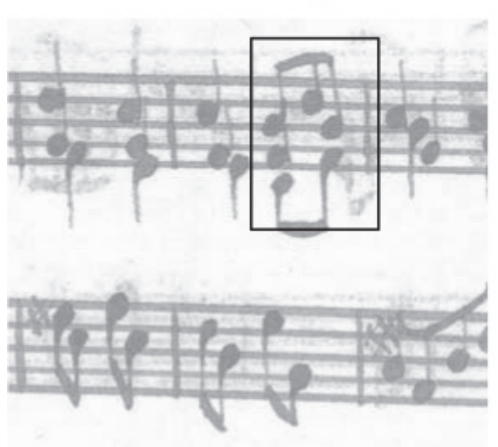

cc. 147 - 148, manuscrito.

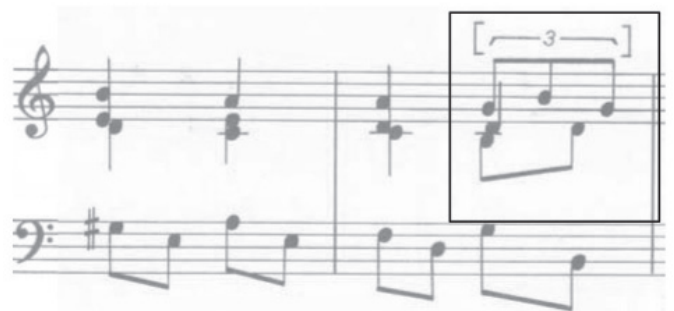

cc. 147 - 148, versión de Ramírez

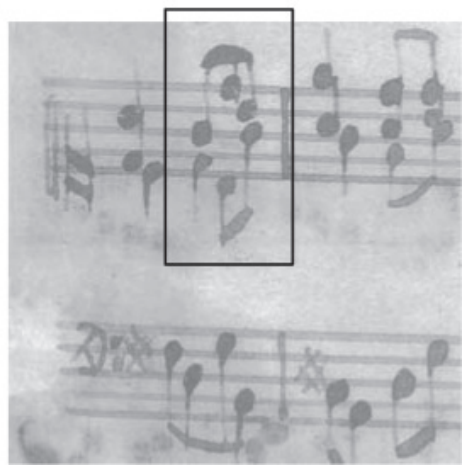

cc. $151-152$, ms.

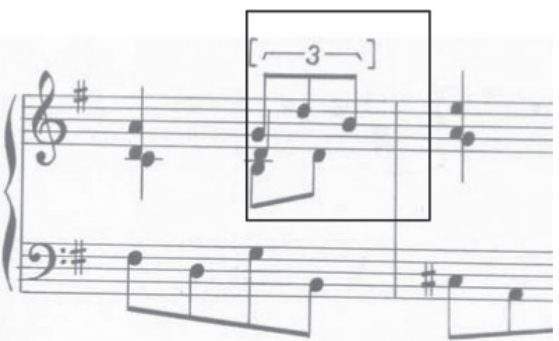

cc. 151 - 152, versión de Ramírez ${ }^{26}$

Mi transcripción:
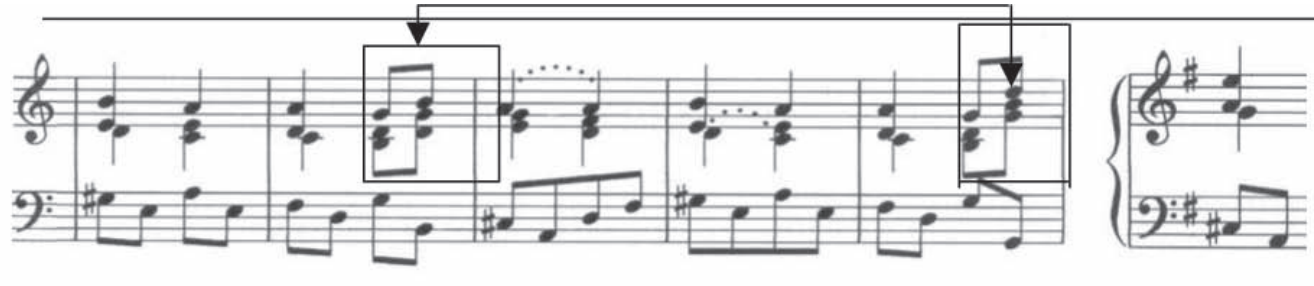
$\begin{array}{lll}6 & 5 & 6 \\ 5 & & 5\end{array}$
6
5

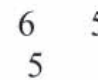
65
6 , etc.
5

cc. 147 - 152 de mi versión

Puede inferirse claramente que todo el pasaje hace referencia a una escritura cordal, básicamente alude a las formas de acompañamiento de un bajo continuo, y en concreto a una secuencia de ligaduras

26 Ramírez, Felipe: El libro que contiene..., op. cit., p. 6, 7, cc. 148 - 151. 
burladas. Justamente los cambios de posición del acorde de Sol, señalados con una flecha, son el punto de intersección de la secuencia, a partir de donde es posible realizar un cambio de posición del acorde de manera "correcta", y pasar a otra región del teclado, sin efectuar faltas en la conducción de las voces.

No hay que dejar de reconocer, que el ms. es de una imprecisión "aparente" a los ojos del lector de música de nuestro tiempo, y, que su interpretación no es nada evidente en relación a nuestros parámetros actuales de concepción musical y gráfica. Podría decir que el Libro de Torres, es una “alegoría de lo aparente". Esta es la fascinación del ms., ya que a través del análisis de cualquiera de sus rubros, ya sea su parte gráfica, aquella de la teoría musical de la época, la elaboración de las ideas musicales, o, su concepción rítmica, etcétera, nos lleva a un mundo de elucubración, de reflexión, en donde las ideas de sus creadores se manifiestan de formas siempre abiertas y multidimensionales. En este sentido, una de las posibilidades que nos permite hacer asequible su interpretación, e incluso su "corrección", radica fundamentalmente en tratar de acertar la mente especulativa y la mente musical de sus creadores. Esta reflexión evidentemente no hace alusión meramente al despliegue gráfico del ms., sino a toda una diversidad de rubros que se analizan a lo largo de este estudio.

El último ejemplo que quiero citar en esta pieza, es también muy elocuente respecto a la modificación extrema que sufren las figuras en la transcripción de Ramírez. Se trata de la última sección, a manera de cláusula final de la "Fuga". El manuscrito presenta la siguiente escritura de los cc. $196-200$ :

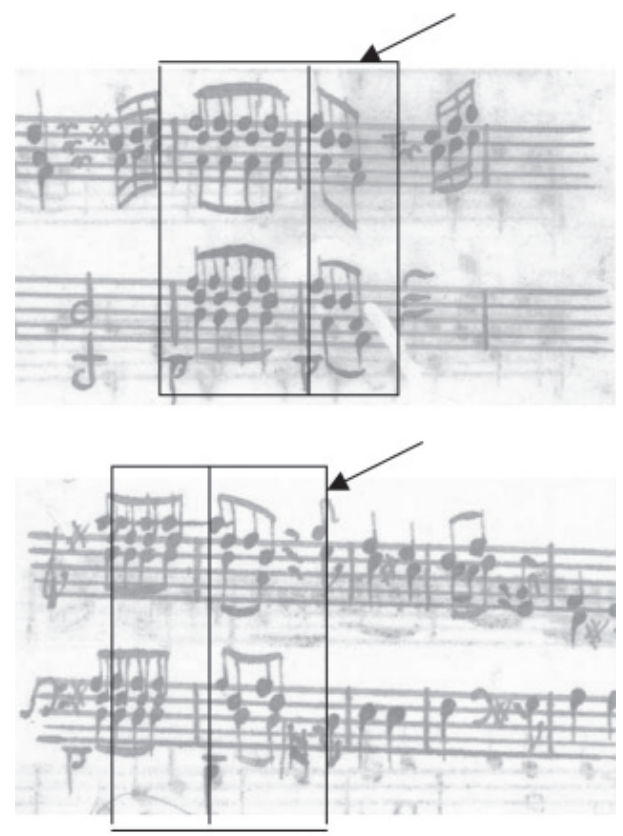

"Fuga, cc. 196 - 202, manuscrito. 
El trabajo de Ramírez presenta la siguiente interpretación:

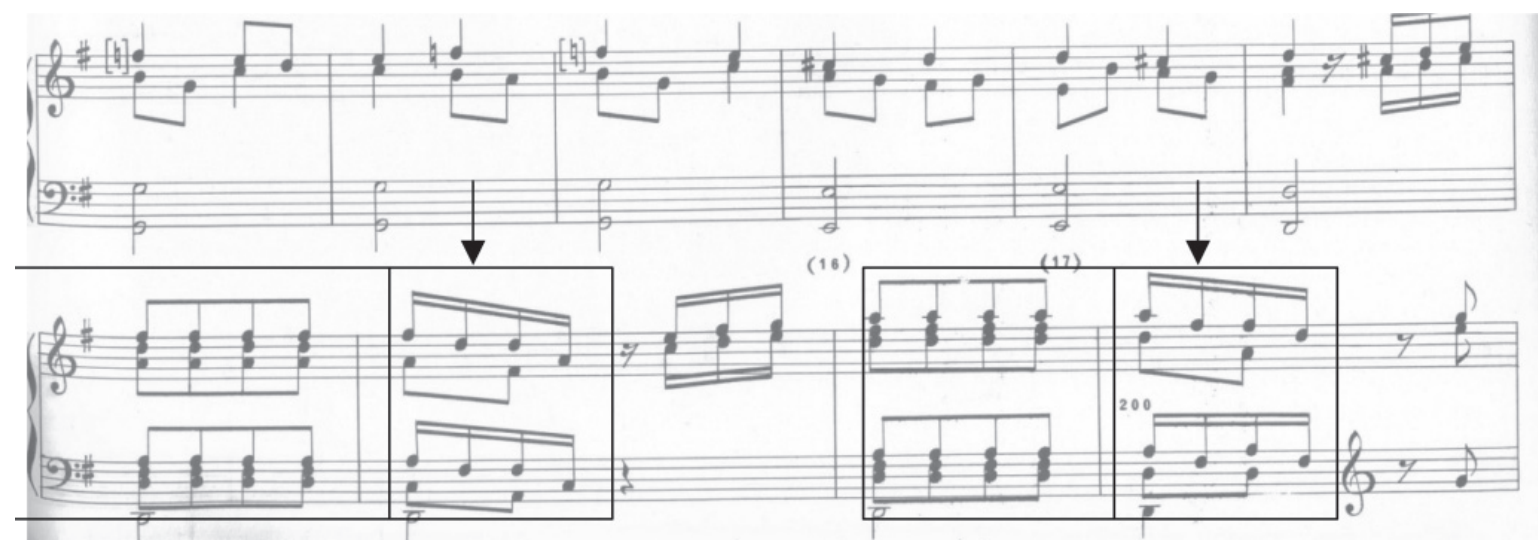

"Fuga", cc. 190 - 200, realización de Ramírez ${ }^{27}$

Mi transcripción:
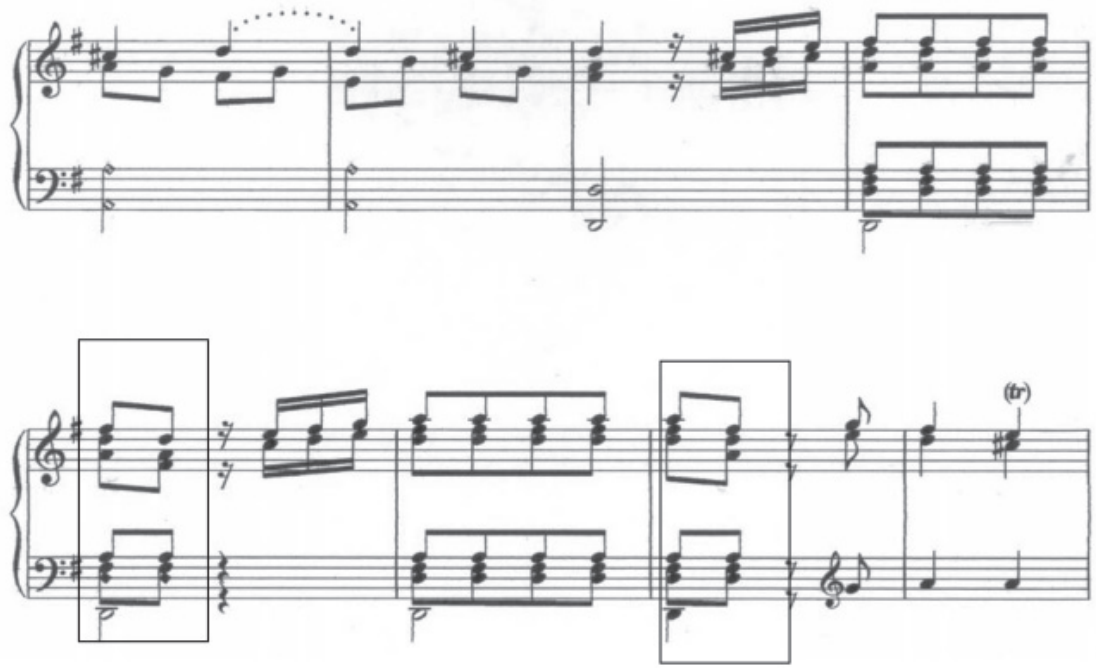

"Fuga", cc. 194 - 201, de mi versión.

27 Ramírez Ramírez, Felipe: El libro que contiene..., op. cit., p. 8, cc. 190 - 200. 


\section{Caso VI. Partido de $2^{\circ}$ Tono.}

Este caso es muy peculiar, ya que nos vuelve a mostrar la interpretación errónea de la información implícita en el ms. por parte de la transcripción de Felipe Ramírez. Sin embargo, también es un nuevo ejemplo, claro y evidente, de la inconsistencia en la realización del manuscrito; es decir, ni la transcripción de Ramírez, ni la versión ofrecida por el manuscrito, son correctas. Pese a ello, el manuscrito. nos da una información hasta cierto punto bastante clara de sus intenciones, las cuales no son restauradas en la edición de Felipe Ramírez. Se trata de los cc. 9 - 15 del "Partido de 2० Tono". Como puede observarse en el siguiente ejemplo, tomado del manuscrito, es evidente el desfase de la mano derecha respecto de la izquierda. Este problema en la escritura genera un desplazamiento de todo un compás, haciendo que el c. 13 de la mano derecha este escrito simultáneamente con el 14 de la mano izquierda. El amanuense, para compensar el problema, escribe un compás de más en la mano izquierda, repitiendo el c. 15 (16 compases contra 15 de la mano derecha para compensar el desfase).

$\begin{array}{lllllll}9 & 10 & 11 & 12 & 13 & 14 & 15\end{array}$

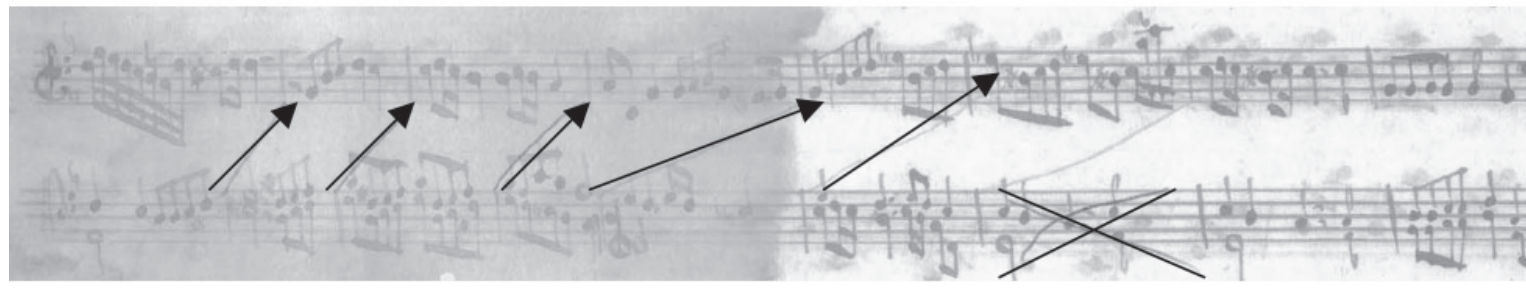

$\begin{array}{llllllll}9 & 10 & 11 & 12 & 13 & 14 & 15 & 16\end{array}$

Ejemplo: cc. 9 a 15 del manuscrito.

Por otro lado, la transcripción de Felipe Ramírez elimina el c. 13 de la mano izquierda (consciente o inconscientemente) para compensar el desfase, en lugar de eliminar el compás 15 o el 16, los cuales se repiten. El resultado de su transcripción es el siguiente:

12

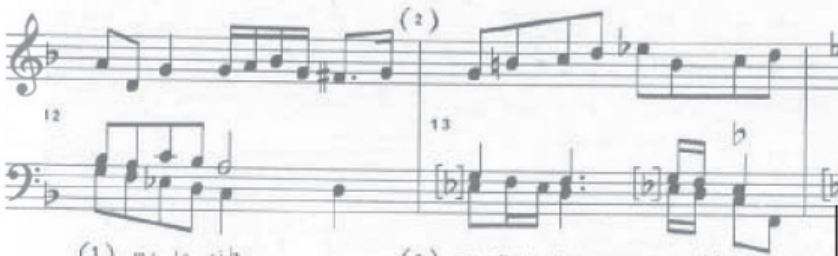

(1) $\mathrm{min}, \mathrm{la}, \mathrm{sib}$.

12
13

(2)

14
14

15

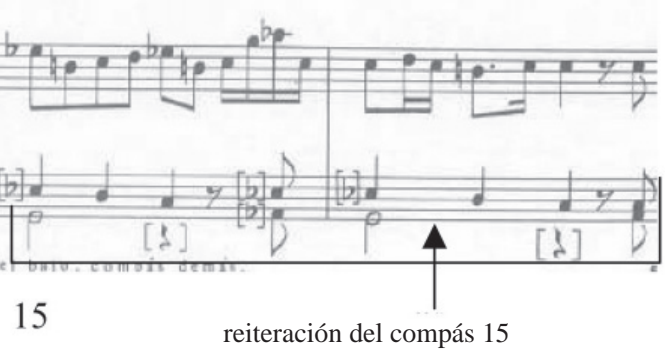

Ejemplo: cc. 12 a 15 de la edición de Ramírez 
En el c. 13, elimina el 13 de la mano izquierda superponiéndole el 14, generando así un desfase de un compás en la mano izquierda respecto de la derecha, y compensándolo con la reiteración del c. 15 que se repite en el manuscrito.

La solución al problema, más allá de suponer que el amanuense simplemente ha repetido el c. 15 en la mano izquierda para compensar el desfase en el manuscrito, radica en observar que se trata de un contrapunto invertible entre sujeto y contrasujeto (en la inversión, el tema en el Tiple pasa al Tenor y el contrasujeto pasa al Tiple), el cual brinda la solución correcta a todas luces, como puede verse en la solución que a continuación propongo.

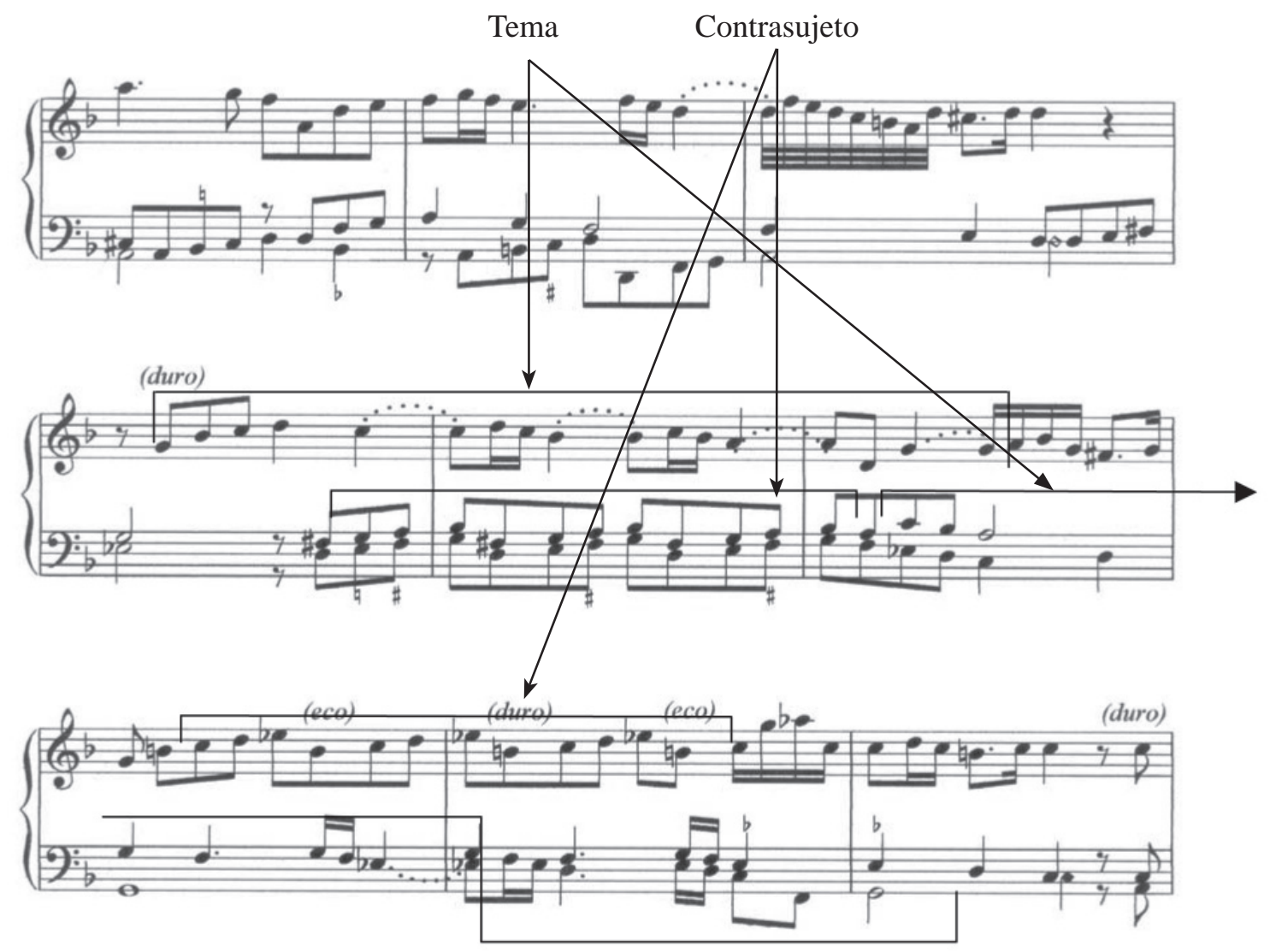

“Partido de $2^{\circ}$ de Torres, cc. 7 - 15, de mi versión

Este ejemplo vuelve a mostrar el grado de experimentación a que estaban sometidos los realizadores del Libro de Torres, ya que podemos observar que el desplazamiento de la escritura musical del ejemplo anterior parece ser una prueba de que la música la están pensando efectivamente en forma horizontal y no en forma vertical (así se hacía y no de otro modo, se estudiaba contrapunto y no "armonía" como 
hoy). De hecho, Torres, en su tratado, es el primero en poner por escrito la concepción vertical/cordal de la música, algo muy nuevo en el ámbito hispánico ${ }^{28}$.

También es de observar que el c. 13 (en el manuscrito) en mano izquierda, muestra una serie de cabildeos del amanuense, gracias al hecho de que en la realización del manuscrito prácticamente no hay correcciones, los errores permanecen, y las enmiendas subsisten al lado de éstos, como puede verse en el ejemplo siguiente:

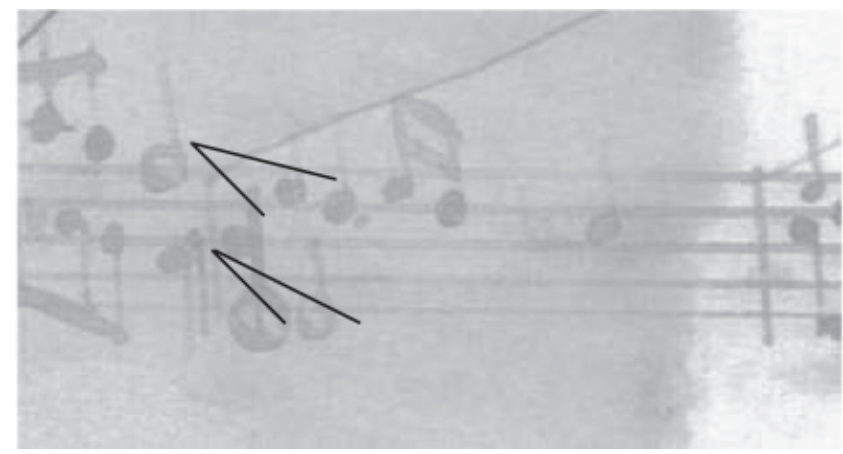

Ejemplo: c. 13

Es evidente el movimiento de quintas paralelas en Tenor y Bajo (Re-La, Sol-Re). Esta solución no es la correcta, razón por la cual los realizadores del Libro de Torres hacen la corrección llevando la quinta Re-La, a la octava Sol-Sol; sin embargo, no borran la primera solución incorrecta, dejando un claro testimonio de la mente especulativa de los realizadores en la búsqueda de diferentes soluciones (!). Como ya he señalado, en este caso, como en muchos otros, los realizadores del manuscrito muestran que no están haciendo una labor de copia, sino de experimentación y aprendizaje de las reglas y teoría de la composición.

\section{USO IDIOMÁTICO DEL TECLADO Y UNIDAD ESTILÍSTICA}

Se ha especulado acerca de la posibilidad de una autoría compartida entre Joseph de Torres y $\operatorname{otros}^{29}$, partiendo de la idea de que la copia manuscrita que nos ocupa se pudo haber compilado en

28 Pedrell, Felipe: P. Antonio Eximeno. Glosario de la gran remoción de ideas que para mejoramiento de la técnica y estética del arte músico ejerció el insigne jesuita valenciano. Ed. Facsímil. Valencia, Librerías "Paris-Valencia", 1992-2002, p. 10. El padre Antonio Eximeno dice en su Glosario: "[Rameau] habría sido un perfecto maestro de su arte si no hubiese escrito el Nuevo sistema de Música Teórica, y la demostración del principio de la armonía. El hacer la armonía simultanea objeto primero de la Música, se opone al sentimiento común de todos los hombres, que sólo adoptan la Música en cuanto sirve para expresar con la modulación las pasiones el ánimo. Para esto no son de absoluta necesidad los sonidos simultáneos."

29 VAlenzuela, Rubén: "El libro para órgano de Joseph de Torres", en Heterofonía, 120-121, (1999). Valenzuela señala: “[...] las seis obras anónimas restantes $(1,4,5,6,7$, y 11) también pueden ser obra de Torres y Martínez Bravo o de uno o más autores no identificados...”. Idem., p. 52. 
diferentes momentos, incluyendo así, obras de otros autores además de Torres. Asimismo, se ha dicho que los estilos encontrados en las composiciones son tan dispares que difícilmente podrían ser de un solo autor.

Una de las tareas de este estudio, es definir si hay o no, unidad en la obra compilada en este documento. A través de un análisis de la técnica compositiva y del tratamiento idiomático del teclado, entre otros factores, he podido encontrar toda una serie relaciones intrínsecas existentes en el conjunto de piezas contenidas en el manuscrito, las cuales son el resultado de la instrumentación de una técnica compositiva común, que se refleja contundentemente en el uso idiomático del teclado y, en general, en las técnicas de composición empleadas. Las relaciones entre las piezas son enormes. Todo ello brinda sólidos argumentos para confirmar que el Libro de Torres fue concebido como una unidad plasmada a partir de las obras para órgano de José de Torres, a quien de hecho se pueden atribuir argumentadamente las obras contenidas en el manuscrito. En este sentido, se puede demostrar que el Libro de Torres no es una recopilación de piezas a la manera de otras colecciones españolas y portuguesas como por ejemplo el Huerto Ameno de Fray Antonio Martín y Coll (†Madrid, 1733c); o El Livro de Obras de Orgao de Diego da Conceicao (1695), en cuyo caso es bien patente la diversidad de autores que las conforman.

Algunas de las relaciones entre las piezas que se analizan a continuación, dan testimonio del enorme sentido de unidad patente en nuestro manuscrito, aunque existan las más diversas referencias e influencias estilísticas a las formas musicales del órgano ibérico de los siglos XVII y XVIII.

Revisemos los siguientes casos:

1- El manejo de la Ligadura de Séptima (Rueda o Círculo, secuencia v - I - V - I).

El empleo de esta ligadura estructurada a partir de una secuencia descendente en el Bajo, es idéntica en su configuración y en su tratamiento idiomático del teclado en numerosas de las obras contenidas en el ms., por ejemplo: en la pieza I ("Fuga"), pieza vi ("Partido de primero Alto"), v ("Obra de primer tono Bajo"), e incluso con un tratamiento idiomático diferente en la pieza VII ("Obra de Torres").

A continuación se muestran los pasajes de las obras mencionadas, en las cuales se manifiestan de manera evidente señales de unidad estilística en el manuscrito (obsérvese también la "progresión" o círculo, o, rueda de quintas en la "Obra de Lleno", compases 66, 67, 68 y 69; esta secuencia es casi idéntica a la progresión de la pieza VI, "Partido 1 Alto", cc. 99 - 100, 101 - 102), de hecho, esta última se realiza sobre los mismos grados. 

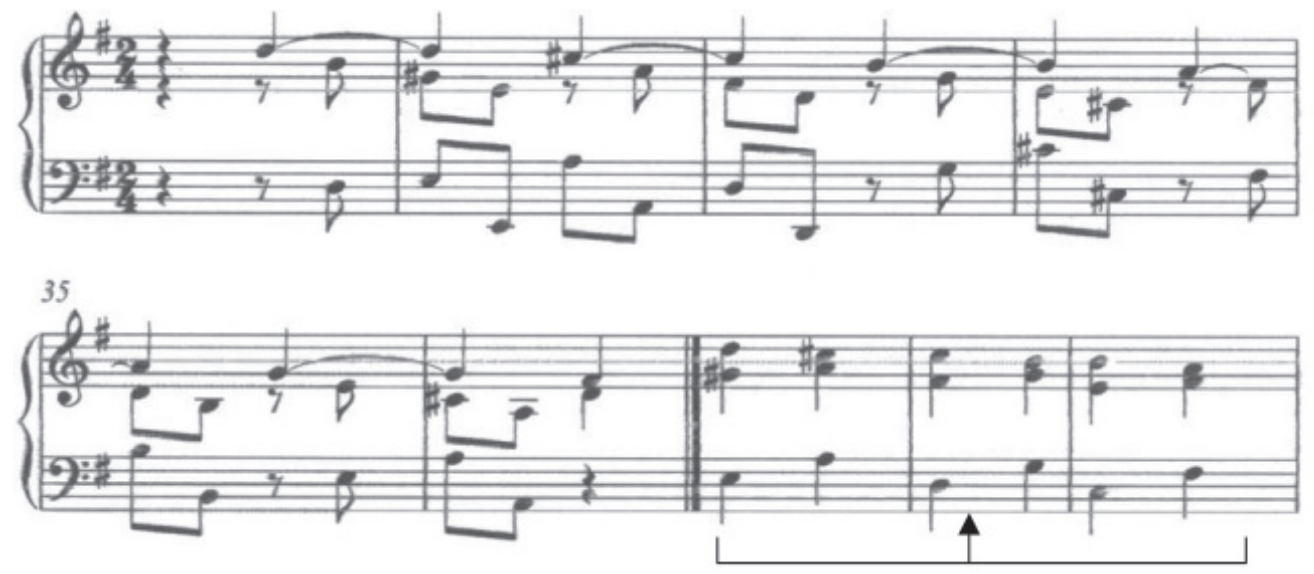

Realización del pasaje en forma cordal

Fragmento de la pieza I, “Fuga”, cc. 87 - 92.

Obsérvese el tratamiento idiomático del movimiento del Bajo a partir de intervalos de saltos de octava. Cotéjese el mismo procedimiento en los siguientes ejemplos, además de mantener siempre la textura a tres voces.

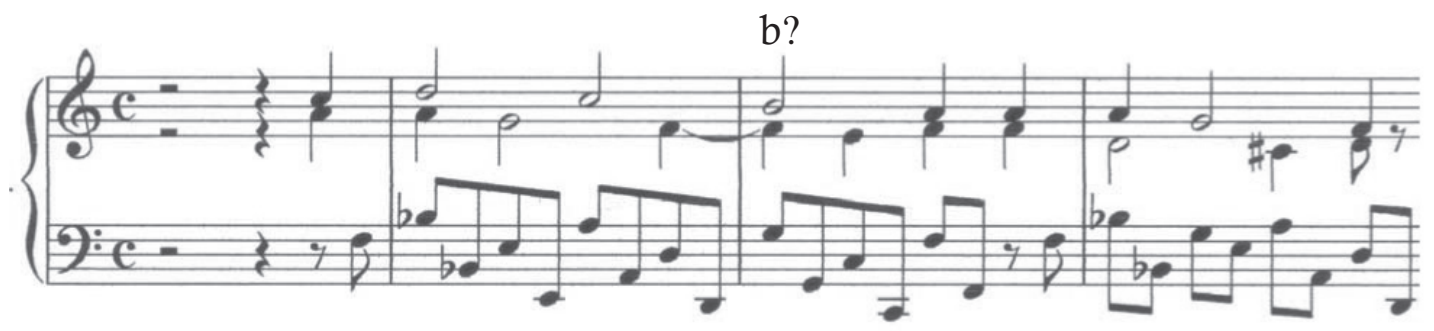

Pieza v, “Obra de 1er Tono bajo”, Ligaduras burladas de séptima, cc. 34 - 37

Cabe señalar que entre ambas piezas existe una clara diferencia de estilo, sin embargo el tratamiento idiomático de este recurso es el mismo en ambos casos; la "Fuga", por ejemplo, está escrita en el estilo de la fuga Italo-Ibérica de mediados del siglo XVIII (esta "Fuga" nos conecta irremediablemente con las fugas de Sebastián Ramón de Albero y Añaños ${ }^{30}$ [*Roncal, Navarra, 10.06.1722; † Madrid, 30.03.1756]). Mientras que la "Obra de $1^{\circ}$ tono Bajo nos hace pensar, de primera intención, en el Tiento de baxón español del siglo XVII y, de manera particular, en aquellos de Pablo Bruna (1611c; †1679).

30 En 1748 fue nombrado primer organista de la Real Capilla. Aparentemente Scarlatti y sus sonatas tuvieron una importante influencia en el estilo de Albero. No deja de llamarnos la atención la presencia de Albero en la Real Capilla, en relación a la posible influencia que también pudo ejercer el mismo Torres en su obra, y de la cual la "Fuga" del Libro de Torres podría ser un buen testimonio. 
También analícese el siguiente ejemplo, tomado de la pieza VI, "Partido de $1^{\circ}$ Alto", en cuyo caso nos encontramos exactamente con el mismo procedimiento idiomático, (de nueva cuenta el salto en octavas, u otras formulas interválicas, a través del círculo o rueda de quintas V - I - V - I, etcétera).

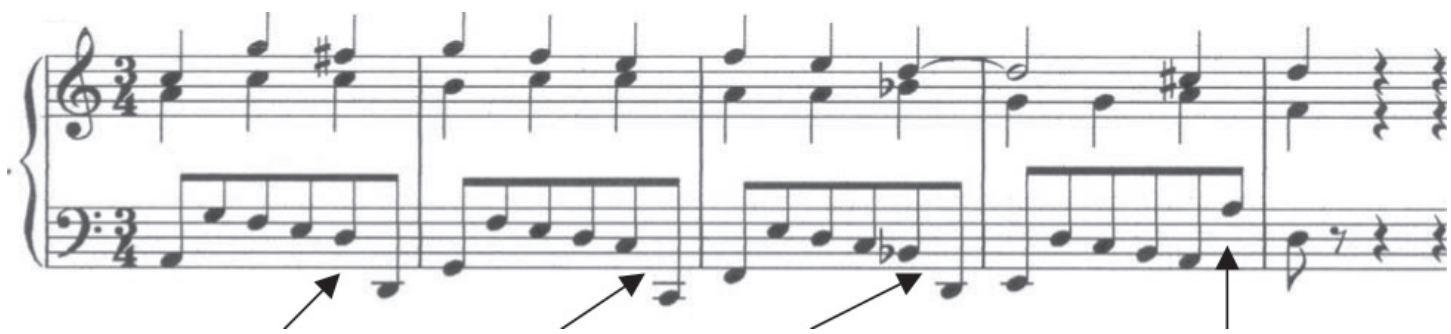

Partido de $1^{\circ}$ alto, cc. 99 - 103

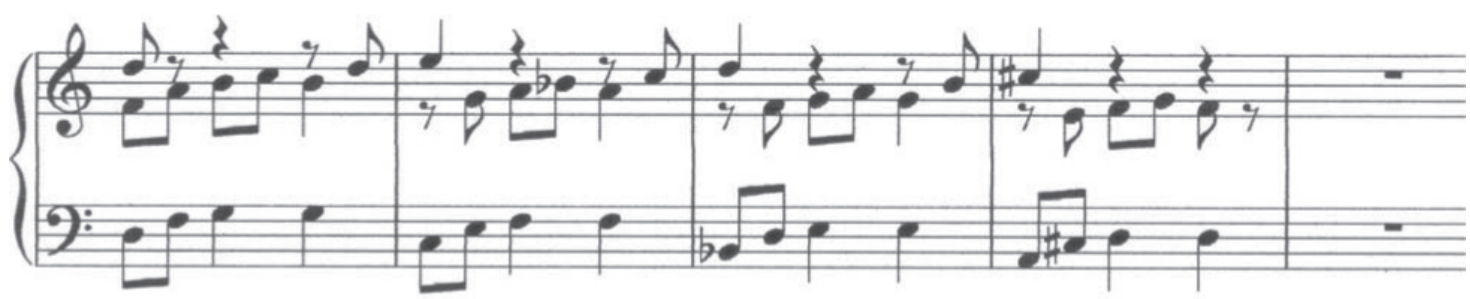

Partido de $1^{\circ}$ alto, cc. $103-106$

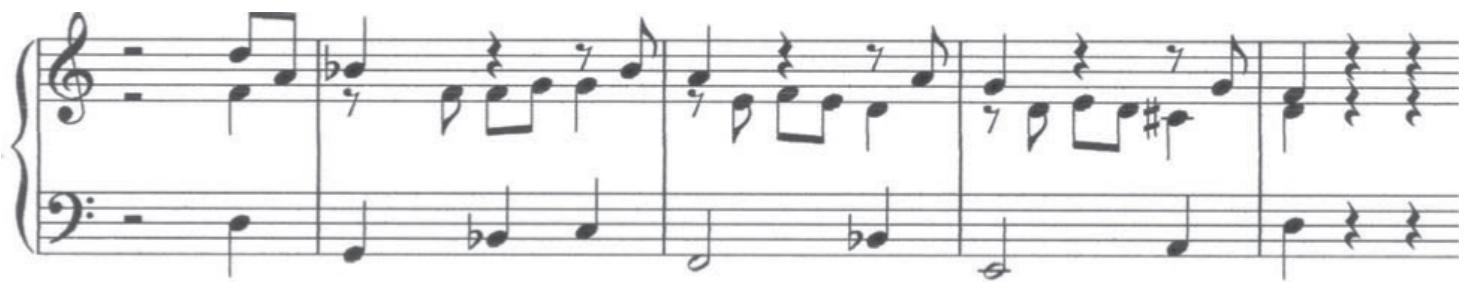

Partido de $1^{\circ}$ alto, cc. $107-109$

El elemento idiomático que caracteriza estas secuencias o "progresiones” en las tres obras precedentes, es el movimiento del Bajo en un carácter netamente instrumental a manera de un bajoncillo, lo cual emparienta este elemento idiomático con el tiento de "medio registro de Baxón”, una de las formas clásicas de la música Ibero-mexicana. En las obras I, v y vi, ya señaladas, el tratamiento de la progresión en círculo o rueda de quintas ${ }^{31}$ se caracteriza no sólo por el movimiento del Bajo en intervalos de cuartas y quintas, sino por el movimiento vigoroso, típicamente instrumental de los intervalos de octava. Este elemento es característico del uso idiomático del teclado y está patente a lo largo de prácticamente toda la colección.

31 En el tratado tercero, capítulo viI, de sus Reglas de Acompañar..., Torres nos dice lo siguiente: "De el Círculo, o Rueda formada con la clausula del baxo. Clausula del baxo, es quando el acompañamiento executa salto de quinta azía abaxo, u de quarta azia arriba, la qual se acompaña cuando es con figuras menores, que no admiten ligadura [...]", op cit., p. 77. 
La "Obra de Torres" (núm. vII), también utiliza marcadamente el recurso del círculo de quintas. En el c. 45 de dicha obra, encontramos nuevamente la progresión 5 6/5, 56/5, 5 6/5.

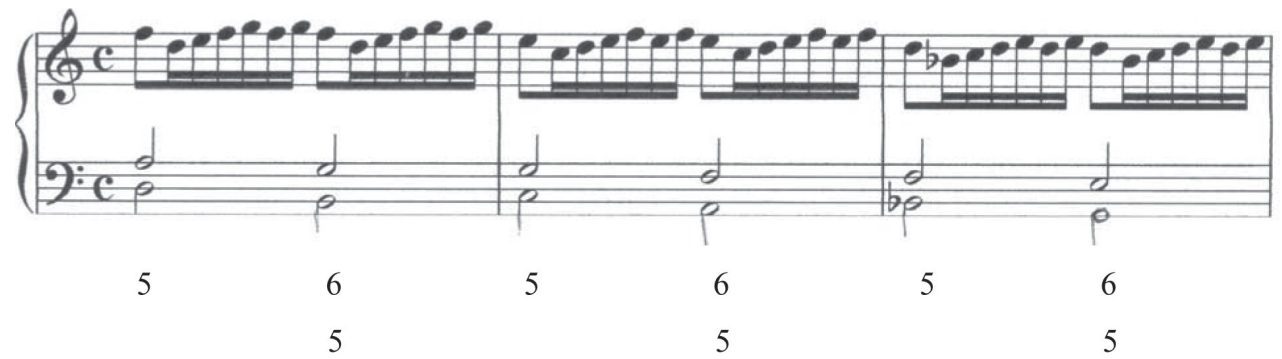

Fragmento de la pieza vII, “Obra de Torres”, cc. 45 - 47.

Se puede observar el mismo tipo de secuencia en movimiento de terceras en el bajo, 7 - 6- 7 - 6, etcétera (básicamente como en el ejemplo anterior), ahora a cuatro voces, en la misma "Obra de Lleno de $7^{\circ}$ tono".

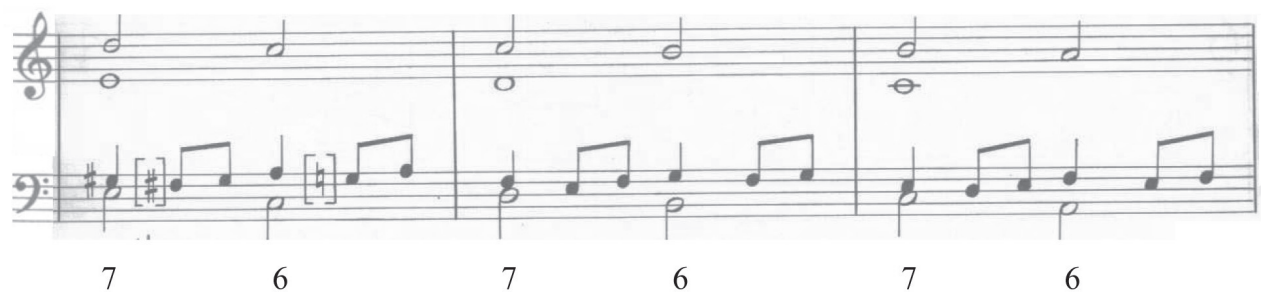

“Obra de Lleno de $7^{\circ}$ tono", cc. 76 a 78.

No obstante lo obvio de la "progresión" iniciada en el c. 45 de la "Obra de Torres" (por terceras en el bajo ${ }^{32}$ ), la transcripción de Felipe Ramírez reincide en escribir los errores del amanuense al transcribir en el c. 45 (3er. tiempo) un La en lugar del Si más que evidente.

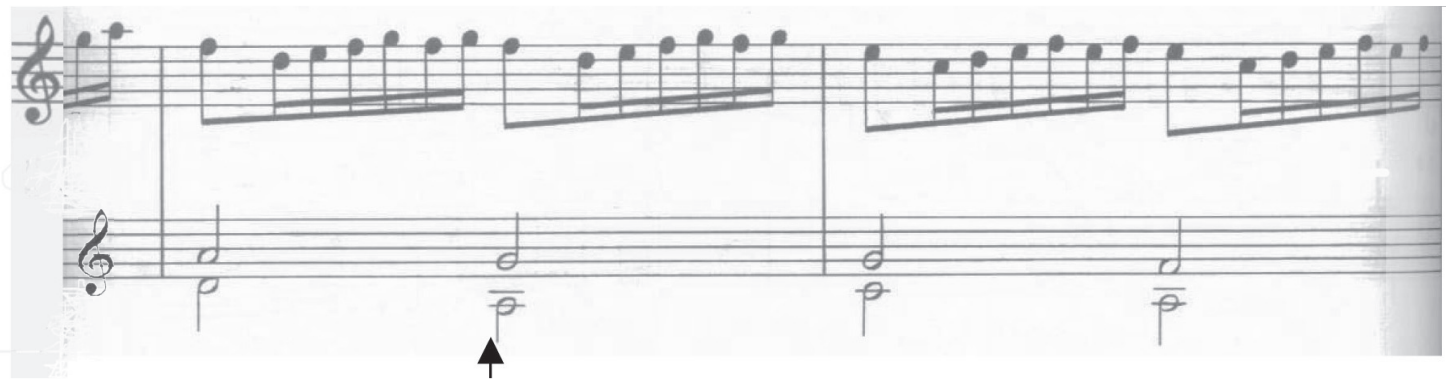

“Obra de Torres”, cc. 45 - 46. Edición de Felipe Ramírez.

32 Se trata de la misma progresión en círculo o rueda de quintas, simplemente se trata de un cambio de postura del acorde. En el caso de la progresión 7-6, el acorde de séptima en estado fundamental resuelve al acorde de sexta. 
Más aún, en el c. 59, encontramos una repetición a la quinta superior de la misma secuencia. En este caso, el amanuense escribió por error clave de Fa en cuarta línea (en el Bajo), cuando en realidad tenía que haber escrito clave de Do en cuarta línea; nuevamente ante la evidencia del error, el transcriptor, simplemente, lo ignora (!).

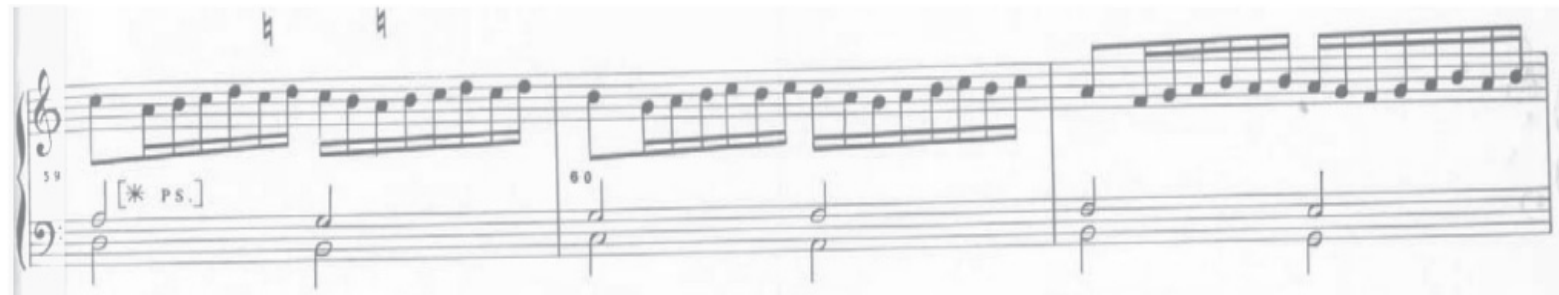

“Obra de Torres”, cc. 59 - 61. Edición de Felipe Ramírez.
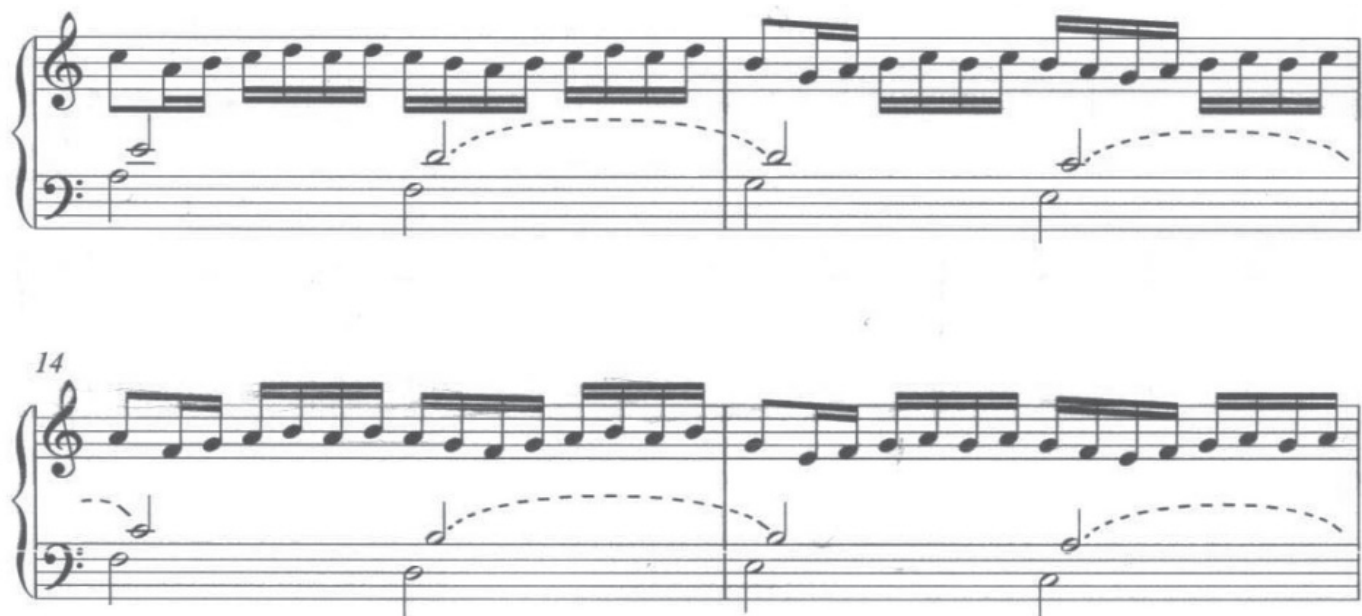

“Obra de Torres”, cc. 59 - 62. Mi versión corregida.

Errores de esta magnitud, hacen completamente inaccesible esta música al intérprete y al auditorio, contribuyendo a que esta música se devalúe intensamente en su contenido técnico y artístico.

El valor documental del Libro de Torres es de gran importancia, y confirma la tesis de que esta colección es un libro de trabajo y práctica de la composición, utilísimo de cara a estudiar cuál era la "técnica de la composición", y de su "enseñanza" entre los organistas de la época.

El Libro de Torres funge como un elemento testimonial de la importancia de la figura de José de Torres en América. Importante compositor de la Corte (también teórico e impresor), adscrito a la Real Capilla, y como tal, "exportable" a otros lugares de la Corona, en este caso México, en donde jugaría - a través de su obra- un destacado papel como "difusor" de modelos en México, lo que habla de su calidad artística y humanística, y de su trascendencia en el mundo hispánico. 


\section{Bibliografía}

Anglés, Higinio: “Orgelmusik der Schola Hispanica von 15. bis 17. Jh.”, en Kgr. Ber. Leipzig, 1926. Reimpreso en Wiesbaden, 1969, pp. 227-231.

APEL, Willi: Geschichte der Orgel- und Klaviermusik bis 1700. Kassel, 1967

Álvarez, José: Colección de obras de órgano de organistas españoles del siglo XVIII. Madrid, UME, 1970.

Bernhard, Christop h: Von der Singekunst oder manier. Kassel: Bärenreiter 1669.

Bach, C. Ph. E.: Versuch über die wahre Art, das Clavier zu spielen. Berlín, Christian Friedrich Henning, (In Verlegong des Autors), 1753.

Bur meist er, Joachim: Hypomnematum Musica Poeticae. Rostock, Stephan Myliander, 1599.

ID.: Musica Poetica. Rostock, Stephan Myliander, 1606.

Delgado Parra, Gustavo; y Gómez Castellanos, Ofelia: Órganos Históricos de Oaxaca. México, Conaculta-Inah, Fomento Cultural Banamex, A.C., 1999.

ID.: "Los Órganos Históricos de la Catedral de México", en Anuario Musical, 60, (2005), pp. 41 - 70.

EzQuerro, Antonio: "El estudio de las marcas de agua del papel como material para determinar la datación y procedencia de las fuentes histórico-musicales, y su grado de fiabilidad", en Anuario Musical, 55, (2000), pp. 19 - 69.

ID.: "Nuevos datos para el estudio de los músicos Nebra en Aragón", en Anuario Musical, 55, (2000), pp. $113-156$.

ID.: "Casos curiosos, peculiaridades y formas alternativas de anotar la música en el área hispánica en el siglo XVII. Procesos de intercambio entre lo culto y lo popular”, en Anuario Musical, 56, (2001), pp. $97-113$.

Galilei, Vincenzo: Dialogo della musica antica e della moderna. Milano: Alessandro Minuziono Editore, 1947.

Jambou, Louis: "Documentos relativos a los músicos de la segunda mitad del siglo XVII de las capillas reales y villa y corte de Madrid, sacados de su Archivo de Protocolos", en Revista de Musicología, XII/2, (1989), pp. 469-514.

Johnson, Calvert: Cuaderno de Tonos y Maitines de Sor María Clara del Santísimo Sacramento. North Carolina, Wayne Leupold Editions, 2005.

Kastner, Macario Santiago: Contribución al estudio de la música española y portuguesa. Lisboa: Atica, 1941.

ID.: "Tres libros desconocidos con música orgánica en las bibliotecas de Oporto y Braga", en Anuario Musical, I, (1946), pp. 143-51.

León Tello, Francisco José: La teoría española de la música en los siglos XVII y XVIII. Madrid, CSIC, 1974.

Levasseur-Rebollo, Yves: Life and Works of José de Torres y Martínez Bravo. Tesis doctoral, U. Pittsburg, 1975.

Listenius, Nikolaus: Musica. Wittemberg, Georg Rhau, 1537. 
Lolo, Begoña: La música en la Real Capilla de Madrid: José de Torres y Martínez Bravo (h16701738). Madrid, U. Autónoma de Madrid, 1990.

ID.: "Felipe v y la música en la corte española entre 1700-1714", en Torre de Lujanes, 38 (1998), pp.107-119.

Lolo, Begoña, Martín P., Peciña C. y Vincent A.: Catálogo del archivo de música del Palacio Real de Madrid. Madrid, Ed. Patrimonio Nacional, 1993.

Lorente, Andrés: El Por qué de la Música. Alcalá de Henares, Nicolás de Xamares, 1672.

Martín y Coll, Fray Antonio: Flores de Música, obras y versos de varios organistas. Año de 1706. E-Mn, M. 1357.

ID.: Pensil deleitoso de suabes flores de música..., 1707. E-Mn, M. 1358.

ID.: Huerto ameno de varias flores de música..., Año de 1708. E-Mn, M. 1359.

ID.: Huerto ameno de varias flores de música..., 1709. E-Mn, M. 1360.

Nassarre, Fray Pablo: Fragmentos Músicos. Madrid, Imprenta Real de Música, 1700.

ID.: Escuela Música según la práctica moderna. Zaragoza, por los Herederos de Diego de Larumbe, 1723-1724.

RAmíRez Ramírez, Felipe, ed: El libro que contiene onze obras para órgano de registros partidos del Dr. D. Joseph de Torres. México, Cenidim, 1993.

ToRres, José de: Reglas Generales de de acompañar, en órgano, clavicordio, y harpa, con solo saber cantar la parte, o un baxo en canto figurado. Madrid, Imprenta de Música, 1702.

ID.: Reglas Generales de acompañar, en órgano, clavicordio, y harpa, con solo saber cantar la parte, o un baxo en canto figurado. Segunda edición. Madrid, Imprenta de Música, 1736.

Zarlino, Gioseffo: Instituzioni Armoniche. Venecia, s.e., 1558.

Recibido: $13 / 10 / 08$

Aceptado: 10/11/08 